\title{
Determination of antineutrino spectra from nuclear reactors
}

\author{
Patrick Huber* \\ Center for Neutrino Physics, Department of Physics, Virginia Tech, Blacksburg, Virginia 24061, USA
}

(Received 16 June 2011; published 29 August 2011)

\begin{abstract}
In this paper we study the effect of well-known higher-order corrections to the allowed $\beta$-decay spectrum on the determination of antineutrino spectra resulting from the decays of fission fragments. In particular, we try to estimate the associated theory errors and find that induced currents like weak magnetism may ultimately limit our ability to improve the current accuracy and under certain circumstance could even greatly increase the theoretical errors. We also perform a critical evaluation of the errors associated with our method to extract the antineutrino spectrum using synthetic $\beta$ spectra. It turns out that a fit using only virtual $\beta$ branches with a judicious choice of the effective nuclear charge provides results with a minimal bias. We apply this method to actual data for ${ }^{235} \mathrm{U},{ }^{239} \mathrm{Pu}$, and ${ }^{241} \mathrm{Pu}$ and confirm, within errors, recent results, which indicate a net $3 \%$ upward shift in energy-averaged antineutrino fluxes. However, we also find significant shape differences which can, in principle, be tested by high-statistics antineutrino data samples.
\end{abstract}

DOI: 10.1103/PhysRevC.84.024617

\section{INTRODUCTION}

Neutrino physics as a branch of experimental physics started with Cowan et al.' s detection of neutrinos produced in a nuclear reactor [1]. Since then, reactor neutrino experiments have played a crucial role in shaping our understanding of the physical properties of the neutrino ${ }^{1}$ (for a review, see, e.g., Ref. [2]), and will continue to do so in the future [3-5]. In a nuclear reactor there are about six $\beta$ decays, and hence neutrinos, per fission or about $2 \times 10^{20}$ neutrinos per second per GW of thermal power. Fortunately, there are only four isotopes whose fissions make up more than $99 \%$ of all reactor neutrinos with an energy above the inverse $\beta$-decay threshold: ${ }^{235} \mathrm{U},{ }^{239} \mathrm{Pu},{ }^{241} \mathrm{Pu}$, and ${ }^{238} \mathrm{U}$. Nonetheless, the resulting neutrino flux is a superposition of thousands of $\beta$-decay branches of the fission fragments of those four isotopes and thus a first-principles calculation is challenging, even with modern nuclear structure data [6]. Therefore, reactor neutrino fluxes from the thermal fission of ${ }^{235} \mathrm{U},{ }^{239} \mathrm{Pu}$, and ${ }^{241} \mathrm{Pu}$ are obtained by inverting measured total $\beta$ spectra which were obtained in the 1980s at the Institut Laue-Langevin (ILL) [7-9]. There are, currently, no corresponding total $\beta$ spectra for ${ }^{238} \mathrm{U}$ since this isotope is fissioned only by fast neutrons. Modern multibaseline experiments [3-5] are designed to be independent of a very precise flux knowledge at the source; however, many of the previous measurements employed only one baseline [2], and initial data from Double Chooz [3] and Daya Bay [4] most likely will be single-baseline data, too.

Recently, a reevaluation of reactor neutrino spectra has been performed [6] and a significant upward shift in the predicted fluxes of about $3 \%$ was found. This in turn translates into a weakening of existing reactor bounds on the absence of $\bar{v}_{e}$ disappearance with wide-ranging consequences for the possible existence of a sterile neutrino with a $\Delta m^{2} \gtrsim 1 \mathrm{eV}^{2}$ $[10,11]$. Here, we present an independent inversion of the ILL

\footnotetext{
*pahuber@vt.edu

${ }^{1}$ Throughout this paper we will refer to both neutrino and antineutrino simply as "neutrino" and rely on the context to avoid ambiguity.
}

PACS number(s): 23.40.Bw, 14.60.Pq, 25.85.Ec, 12.60.-i

$\beta$ spectra into neutrino spectra for the isotopes ${ }^{235} \mathrm{U},{ }^{239} \mathrm{Pu}$, and ${ }^{241} \mathrm{Pu}$. To this end, we first review various corrections to the allowed $\beta$-decay shape and estimate the associated theory errors in Sec. IID. We use virtual $\beta$ branches and apply the corrections obtained in Sec. II D individually for each branch. We then use synthetic data sets and Monte Carlo simulations in Sec. III A to quantify the bias and statistical errors associated with the inversion procedure. In Sec. III B we compute the effective nuclear charge $\bar{Z}$ and provide error estimates. We then apply the method that we have developed to the actual $\beta$ data and present our result on the neutrino fluxes in Sec. IV. In Sec. $\mathrm{V}$ we perform a critical comparison of our result with previously obtained neutrino fluxes and point out a possible explanation for the flux shift in terms of known nuclear physics. Finally, we conclude in Sec. VI.

\section{II. $\beta$ SPECTRA}

In this section we describe corrections to the allowed $\beta$-spectrum shape and provide an estimate of the associated uncertainty. Here, we mainly follow the notation of Ref. [12]. The allowed $\beta$ spectrum is given by [13]

$$
N_{\beta}(W)=K \underbrace{p^{2}\left(W-W_{0}\right)^{2}}_{\text {phase space }} F(Z, W),
$$

where $W=E /\left(m_{e} c^{2}\right)+1$ and $W_{0}$ is the value of $W$ at the end point. $K$ is a normalization constant. $F(Z, W)$ is the so-called Fermi function and is given by

$$
\begin{gathered}
F(Z, W)=2(\gamma+1)(2 p R)^{2(\gamma-1)} e^{\pi \alpha Z W / p} \frac{|\Gamma(\gamma+i \alpha Z W / p)|^{2}}{\Gamma(2 \gamma+1)^{2}} \\
\text { with } \gamma=\sqrt{1-(\alpha Z)^{2}},
\end{gathered}
$$

where $R$ is the nuclear radius, which in itself has a dependence on $A$. The Fermi function accounts for the fact that the outgoing electron is moving in the Coulomb field of the nucleus and is derived from the solution of the Dirac equation for a pointlike 

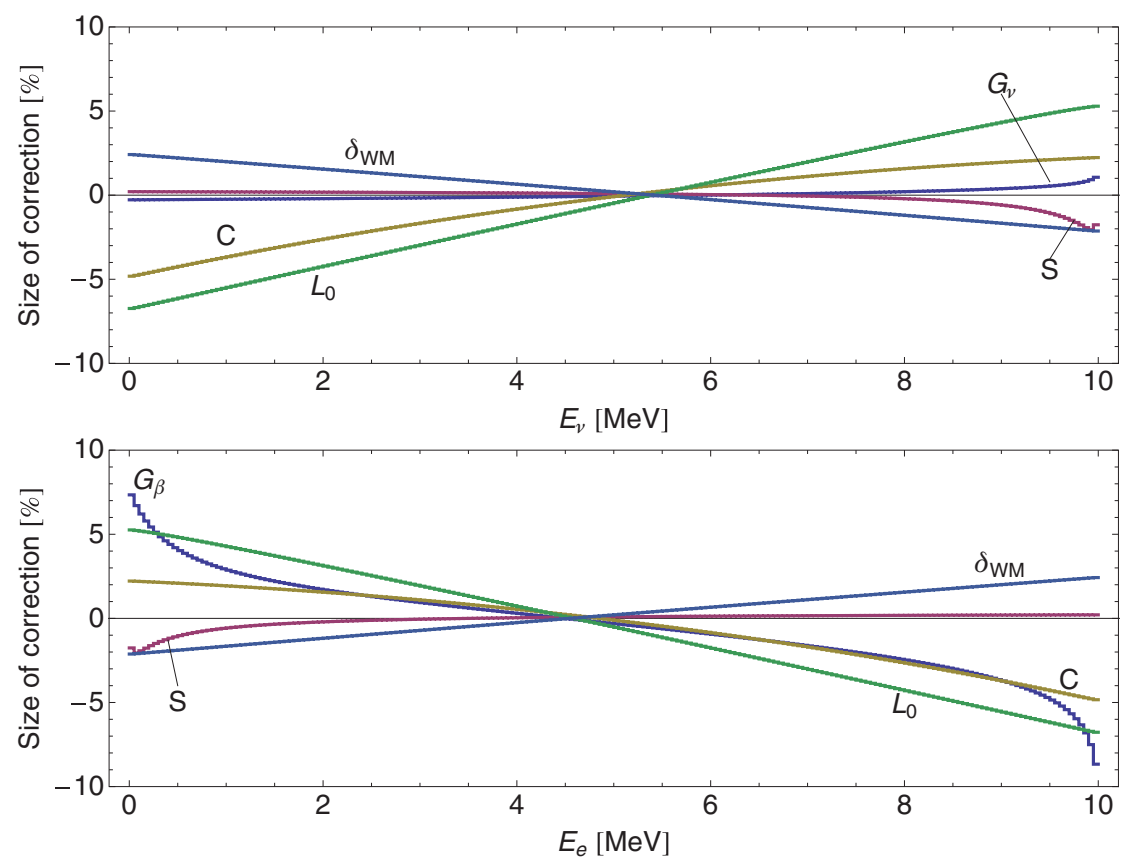

FIG. 1. (Color online) Relative size of the various corrections listed in Eq. (4) for a hypothetical $\beta$ decay with $Z=46, A=117$, and $E_{0}=10 \mathrm{MeV}$. The top panel shows the effect on the neutrino spectrum, whereas the bottom panel shows the effect on the $\beta$ spectrum. and infinitely heavy nucleus. The Fermi function is the leadingorder QED correction to nuclear $\beta$ decay.

In Eq. (1) the $\beta$-spectrum shape for allowed decays is given; however, fission fragments contain a significant fraction of decay branches of unique and nonunique forbidden type. For forbidden decays the simple phase space factor $p^{2}\left(W-W_{0}\right)^{2}$ is multiplied by [14]

$p_{v}^{2}+p^{2}, \quad$ first unique, second nonunique, $p_{v}^{4}+\frac{10}{3} p_{v}^{2} p^{2}+p^{4}, \quad$ second unique, third nonunique, $p_{v}^{6}+7 p_{\nu}^{4} p^{2}+7 p^{4} p_{\nu}^{2}+p^{6}, \quad$ third unique, fourth nonunique.

We see that the forbidden space phase factor is symmetric under the exchange of $p$ and $p_{v}$ and thus, if we had $m_{v}=m_{e}$, the whole expression would be symmetric between neutrino and electron energies, too. If we keep the measured $\beta$ spectrum fixed and fit a $\beta$ shape to it, any change to the $\beta$ shape that is symmetric between $E_{e}$ and $E_{v}$ would not change the neutrino spectrum. This is why the corrections from forbidden decays to inverted neutrino spectra have been found to be small [6-9], despite the overall large contribution of forbidden decays in fission fragments.

It turns out that for precision studies a number of additional effects have to be taken into account, and the modified $\beta$ spectrum becomes [15]

$$
\begin{aligned}
N_{\beta}(W)= & K p^{2}\left(W-W_{0}\right)^{2} F(Z, W) L_{0}(Z, W) \\
& \times C(Z, W) S(Z, W) G_{\beta}(Z, W)\left(1+\delta_{\mathrm{WM}} W\right) .
\end{aligned}
$$

The neutrino spectrum is obtained by the replacements $W \rightarrow$ $W_{0}-W$ and $G_{\beta} \rightarrow G_{\nu}$. In the following we describe the physical origin and the actual expression used for each of these additional terms. The size of the contribution of each of these terms for a typical $\beta$ decay is shown in Fig. 1.

\section{A. Finite-size corrections}

There are two effects from the finite size of the nucleus: the electric charge distribution is no longer pointlike and the hypercharge distribution is no longer pointlike, i.e., the nucleon is moving inside the nuclear potential. For all finite-size corrections we need to be able to determine the nuclear radius ${ }^{2}$ as a function of $A$ and we use the so-called Elton formula [17]

$$
R=0.0029 A^{1 / 3}+0.0063 A^{-1 / 3}-0.017 A^{-1}
$$

in units of $m_{e} c^{2}$.

The electromagnetic finite-size effect is expressed by $L_{0}$, and we use the approximation given in Ref. [15]:

$$
\begin{aligned}
L_{0}(Z, W)= & +13 \frac{(\alpha Z)^{2}}{60}-W R \alpha Z \frac{41-26 \gamma}{15(2 \gamma-1)} \\
& -\alpha Z R \gamma \frac{17-2 \gamma}{30 W(2 \gamma-1)}+a_{-1} \frac{R}{W} \\
& +\sum_{n=0}^{5} a_{n}(W R)^{n}+0.41(R-0.0164)(\alpha Z)^{4.5}
\end{aligned}
$$

where the $a_{n}$ are given by

$$
a_{n}=\sum_{x=1}^{6} b_{x}(\alpha Z)^{x},
$$

and the $b_{x}$ are taken from Table I of Ref. [15] and reproduced here in Table V in Appendix A. We checked that the error of this

${ }^{2}$ Differences due to different shapes of the charge distribution, e.g., constant within the nucleus or all charge on the surface, are very small and can be neglected; see, e.g., [16]. Also, reasonable variations of the Elton formula have only a minor impact on our result, i.e., a variation of $10 \%$ in $A$ will change the total flux by $0.05 \%$. 
approximation in comparison with numerically exact results given in [14] is smaller than $10^{-5}$. Neglecting the last three terms in the above expression for $L_{0}$ still produces accurate results, and this form of $L_{0}$ produces results very close to the ones summarized as finite-size effects in Eq. (8) of Ref. [6].

The weak-interaction finite-size correction is of the following form for Gamow-Teller decays ${ }^{3}$ [15]:

$$
\begin{aligned}
C(Z, W) & =1+C_{0}+C_{1} W+C_{2} W^{2} \text { with } \\
C_{0} & =-\frac{233}{630}(\alpha Z)^{2}-\frac{\left(W_{0} R\right)^{2}}{5}+\frac{2}{35} W_{0} R \alpha Z, \\
C_{1} & =-\frac{21}{35} R \alpha Z+\frac{4}{9} W_{0} R^{2}, \\
C_{2} & =-\frac{4}{9} R^{2} .
\end{aligned}
$$

In Ref. [18] it is estimated that the errors on both these corrections, $L_{0}$ and $C$, are smaller than $10 \%$ of their size. Thus, we can neglect this as a relevant source of error.

\section{B. Screening correction}

$S(Z, W)$ accounts for screening of the nuclear charge by all the electrons in the atomic bound state and it effectively reduces the charge "seen" by the outgoing electron. Our description of the screening correction $S(Z, W)$ follows Ref. [16], and we introduce the following quantities:

$$
\begin{aligned}
& \bar{W}=W-V_{0}, \quad \bar{p}=\sqrt{\bar{W}^{2}-1}, \quad y=\frac{\alpha Z W}{p}, \\
& \bar{y}=\frac{\alpha Z \bar{W}}{\bar{p}}, \quad \tilde{Z}=Z-1 .
\end{aligned}
$$

$V_{0}$ is the so-called screening potential and derived from numerical calculations and can be parametrized as

$$
V_{0}=\alpha^{2} \tilde{Z}^{4 / 3} N(\tilde{Z}),
$$

and $N(\tilde{Z})$ is taken as a linear interpolation of the values in Table 4.7 of Ref. [16], which is reproduced here in Table VI in Appendix A. Then, we obtain

$$
\begin{aligned}
& S(Z, W)=\frac{\bar{W}}{W}\left(\frac{\bar{p}}{p}\right)^{(2 \gamma-1)} e^{\pi(\bar{y}-y)} \frac{|\Gamma(\gamma+i \bar{y})|^{2}}{\Gamma(2 \gamma+1)^{2}} \\
& \text { for } W>V_{0},
\end{aligned}
$$

and $S(Z, W)=1$ for $W<V_{0}$. As can be seen from Fig. 1 , the effect of the screening correction itself is rather small and the theory behind it is well understood [16]; therefore we will not regard it as a source of theoretical errors.

\section{Radiative corrections}

Radiative corrections are due to the emission of virtual and real photons by the charged particles present in $\beta$ decay, and it is crucial to correctly account for both the virtual and real photon contributions to ensure the proper cancellation of

\footnotetext{
${ }^{3}$ Almost all decays relevant in our problem are of this type and the corresponding expression for Fermi decays is numerically very close.
}

divergences. The finite part of the virtual photon exchange contribution does depend on the details of nuclear structure, but affects only the decay width and not the spectral shape. Therefore, the shape effect can be accurately computed. In our presentation we will suppress all terms that affect only the decay width, i.e., are independent of energy, and keep only the energy-dependent parts. The radiative correction at order $\alpha$ has been computed by Sirlin [19] for the $\beta$ spectrum, with $\beta=p / W$,

$$
\begin{aligned}
g_{\beta}= & 3 \ln M_{N}-\frac{3}{4}+4\left(\frac{\tanh ^{-1} \beta}{\beta}\right) \\
& \times\left(\frac{W_{0}-W}{3 W}-\frac{3}{2}+\ln \left[2\left(W_{0}-W\right)\right]\right)+\frac{4}{\beta} L\left(\frac{2 \beta}{1+\beta}\right) \\
& +\frac{1}{\beta} \tanh ^{-1} \beta\left(2\left(1+\beta^{2}\right)+\frac{\left(W_{0}-W\right)^{2}}{6 W^{2}}-4 \tanh ^{-1} \beta\right),
\end{aligned}
$$

where $L(x)$ is the Spence function, defined as $L(x)=$ $\int_{0}^{x} d t / t \ln (1-t)$. The complete correction is then given by

$$
G_{\beta}(Z, W)=1+\frac{\alpha}{2 \pi} g_{\beta}
$$

The radiative correction for neutrinos, $G_{v}$, has been derived in Ref. [20], and we apply the form given in Ref. [20] to the neutrino spectrum. The expressions are somewhat lengthy and involve integrals that cannot be solved in closed form, and therefore we do not quote them here. More recently, the radiative correction for neutrinos has been derived in closed form by Sirlin [21], ${ }^{4}$ and we find that the energy dependence of the new calculation is numerically within $5 \%$ of the previous one [20]. Because all spectra are normalized to unity, differences in terms that do not depend on energy will cancel. Thus. for our purposes the two results are equivalent and since the new result [21] is rather compact, we quote it here:

$$
\begin{aligned}
h_{v}= & 3 \ln M_{N}+\frac{23}{4}-\frac{8}{\hat{\beta}} L\left(\frac{2 \hat{\beta}}{1+\hat{\beta}}\right) \\
& +8\left(\frac{\tanh ^{-1} \hat{\beta}}{\hat{\beta}}-1\right) \ln (2 \hat{W} \hat{\beta}) \\
& +4 \frac{\tanh ^{-1} \hat{\beta}}{\beta}\left(\frac{7+3 \hat{\beta}^{2}}{8}-2 \tanh ^{-1} \beta\right),
\end{aligned}
$$

where $\hat{\beta}=\hat{p} / \hat{E}$ and $\hat{p}=\sqrt{\hat{W}^{2}-1}$ and $\hat{W}=W_{0}-W_{\nu}$, where $W_{v}$ is the energy of the neutrino. The neutrino radiative correction then becomes $G_{v}\left(Z, W_{v}\right)=1+\alpha /(2 \pi) h_{v}$. Note that the radiative correction in the $\beta$ spectrum is quite large, whereas the correction for the neutrino spectrum is very much smaller.

\footnotetext{
${ }^{4}$ I would like to thank T. Schwetz-Mangold for bringing this new work to my attention.
} 


\section{Weak magnetism}

The term "weak magnetism" was coined by Gell-Mann and he proposed it as a sensitive test of the then emerging $\mathrm{V}$-A theory of weak interactions [22]. The weak-magnetism contribution to $\beta$ decay belongs to the class of so-called induced currents, i.e., currents that do not correspond to couplings present in the initial Hamiltonian, and they appear only at finite momentum transfer. Out of these induced currents, the weak-magnetism term typically yields the largest contribution to the shape of the $\beta$ spectrum (see, e.g., [23]), and therefore we will only discuss this term. The effect of weak magnetism is to modify the spectral shape of $\beta$ decay by a factor

$$
1+\delta_{\mathrm{WM}} W \quad \text { with } \quad \delta_{\mathrm{WM}}=\frac{4}{3} \underbrace{\frac{b}{M c}}_{=w} m_{e},
$$

where $c$ is the Gamow-Teller matrix element, $b=\sqrt{2} \mu$, with $\mu$ the magnetic transition moment, and $M=A M_{N}$ is the mass of the nucleus. Experimentally, the transition magnetic moment or $b$ can be determined by measuring the magnetic dipole $M 1$ $\gamma$ decay width $\Gamma_{M 1}$ of the corresponding isovector transition of the isobaric analog state, and $b$ is then given by

$$
b=\left(\frac{6 \Gamma_{M 1} M^{2}}{\alpha E_{\gamma}^{3}}\right)^{1 / 2} \text {. }
$$

This derivation relies on the concept of conserved vector currents (CVCs), which is essentially a necessary result of gauge invariance. In the so-called impulse approximation, one further assumes that the transition magnetic moment is entirely given by the intrinsic anomalous magnetic moments of the proton, $\mu_{p}$, and neutron, $\mu_{n}$, and one obtains for $w$ the simple expression

$$
w \simeq \frac{\mu_{p}-\mu_{n}}{M_{N}}\left|\frac{C_{V}}{C_{A}}\right| .
$$

Note that, in general, there also would be a contribution to $b$ (or $\mu$ ) from the orbital angular momentum of the decaying nucleon, which we neglect here. Within the impulse approximation and neglecting orbital angular momentum, ${ }^{5}$ there is no dependence on nuclear structure and therefore $w$ has the same value for all Gamow-Teller $\beta$ decays. This approximation is the basis for the statement in Ref. [24] that $\frac{d N}{d E}=\frac{4}{3} w=0.5 \% \mathrm{MeV}^{-1}$ is the universal value for the weak-magnetism slope parameter in all $\beta$ decays. The validity of the necessary approximation seems to be supported by the measured value for $\frac{d N}{d E}$ in the $A=12$ isotriplet of ${ }^{12} \mathrm{~B},{ }^{12} \mathrm{C}$, and ${ }^{12} \mathrm{~N}$ [25].

At the same time, one can use the CVC hypothesis and use Eq. (17) to infer the weak-magnetism slope parameter for a number of isotopes from their measured $\gamma$-decay parameters. In Ref. [26] this analysis has been performed on a set of 12 nuclei and a large variation of both $\frac{d N}{d E}$ and $b$ was found;

\footnotetext{
${ }^{5}$ Under these assumptions, there is also no contribution of the weak electric form factor, called $d$ in the notation of [23], which could be sizable.
}

see Table II of Ref. [26]. In Table I we present a somewhat enlarged set of nuclei for which we could identify the necessary information from the Evaluated Nuclear Structure Data File (ENSDF) database [27]. The analysis follows the one outlined in Ref. [26]. For some of the nuclei we find slightly different results in comparison to Ref. [26], which can be traced to changes in the nuclear database, and we have, in all cases, provided the corresponding reference.

This sample of nuclei is small, 16 nuclei only, and the majority are much lighter than typical fission fragments, which raises the issue of how representative this sample is for fission fragments. On the other hand, these nuclei summarize our current experimental understanding of weak magnetism. Setting these doubts aside, we can compute the average value of $\frac{d N}{d E}$ and its standard deviation as a measure of the error. In Table I we have separated four isotopes based on their unusually large $\log f t$ values. In those cases, the Gamow-Teller matrix element is very small and thus the relative size of the weak-magnetism contribution is enhanced. As a result, all of these four isotopes have large values of $\frac{d N}{d E}$. Obviously, these four large-log $f t$ isotopes will completely dominate the average and standard deviation and therefore here the question becomes acute of how representative our sample is for fission fragments. For the main analysis presented in this paper, we therefore exclude these four isotopes, and this yields the following mean and standard deviation:

$$
\frac{d N}{d E}=(0.48 \pm 0.47) \% \mathrm{MeV}^{-1},
$$

which essentially is identical to the value obtained from using the impulse approximation, but we also see that there is significant variance. We will use the standard value of Ref. [24] and assign a $100 \%$ error to it.

If we include the large-log $f t$ isotopes, the corresponding result is

$$
\frac{d N}{d E}=(4.8 \pm 10.9) \% \mathrm{MeV}^{-1},
$$

that is, a ten times larger mean with a twice larger relative error. A shift of $\frac{d N}{d E}$ by $+0.5 \% \mathrm{MeV}^{-1}$ causes a shift of the neutrino rate of about $-1 \%$. Thus, if one were to use Eq. (20) the resulting rate uncertainty would be about $20 \%$ and the neutrino flux shift found in Ref. [6] would be quite easily absorbed in this error bar. Even just a shift of the central value is sufficient to bring the neutrino fluxes nearly back to the original level. Obviously, large values of $\log f t$ imply relatively long half-lives and long-lived isotopes play only a small role in neutrino emission ${ }^{6}$ from fission fragments. However, if the end point energy is large, the relevant Gamow-Teller matrix element can be quite small without leading to a very long lifetime. Also, it is not clear how large weak magnetism would be in forbidden decays, since there the leading-order nuclear matrix element is already quite small and thus all corrections tend to have relatively more weight. Thus, the size of weak magnetism, or more generally induced currents, is the one major source of theory uncertainty. In summary, the reactor anomaly [10] can be attributed either to new physics in the

\footnotetext{
${ }^{6}$ That is, above the inverse $\beta$-decay threshold.
} 
TABLE I. Gamow-Teller decays and the associated parameters needed for a computation of the weak-magnetism slope parameter using the CVC hypothesis.

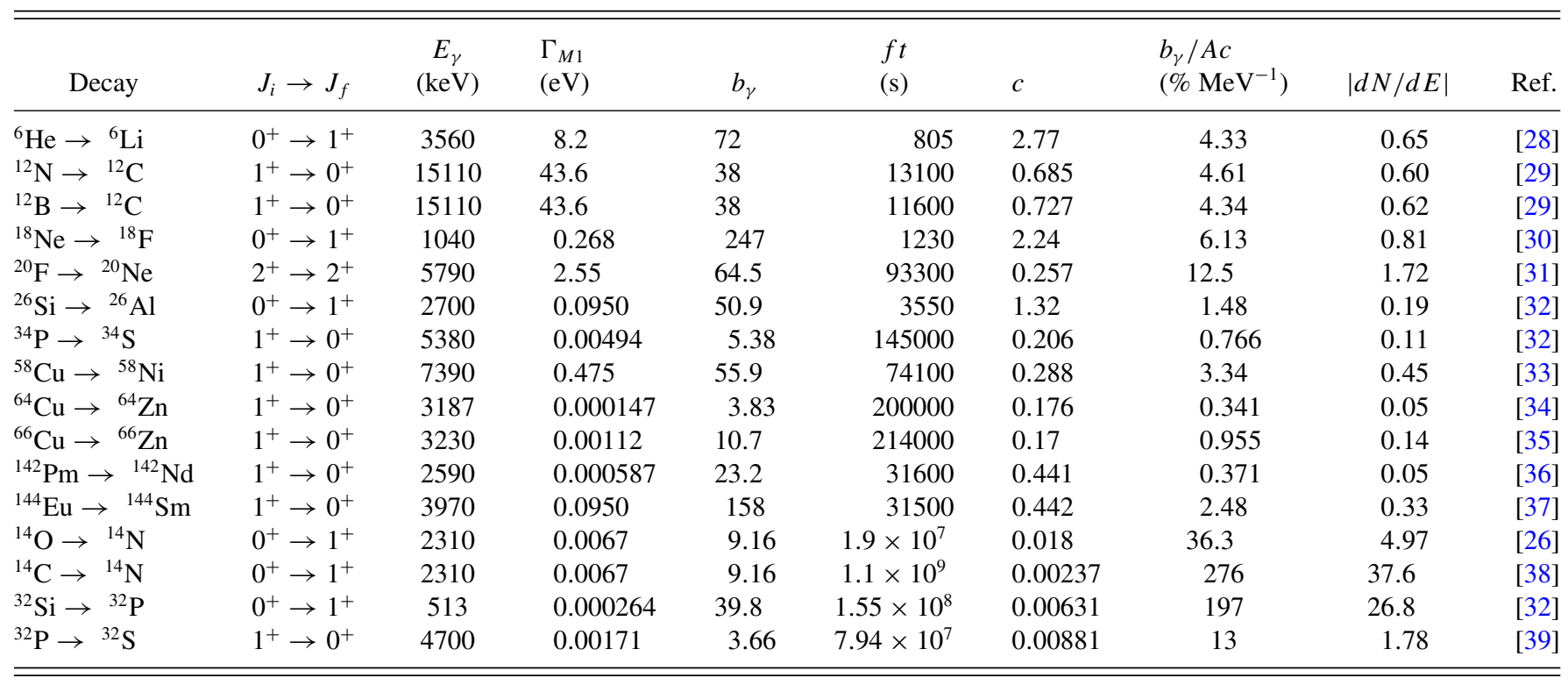

form of a sterile neutrino or to a significant extent to some not well understood nuclear physics. In particular, a detailed study of the breakdown of the impulse approximation in large-log $f t$ nuclei in the relevant mass range would be quite helpful, but is beyond the scope of this paper.

\section{EXTRACTION OF THE ASSOCIATED NEUTRINO SPECTRUM}

For a single $\beta$-decay branch the inversion from the $\beta$ spectrum into the corresponding neutrino spectrum is straightforward due to energy conservation, if one neglects recoil effects, which are $\mathcal{O}\left(E_{0} /\left(A M_{N}\right)\right) \sim 10^{-4}$. In particular, if the $\beta$ spectrum has been measured with good precision, the neutrino spectrum can be known to the same level of precision without any reliance on theory. In practice, however, most of the nuclei involved have many $\beta$ branches, most of which are identified by $\gamma$-ray spectroscopy and not by direct measurement of the $\beta$ spectrum; in these cases, $\beta$-decay theory as outlined in Sec. II plays a crucial role in obtaining the associated neutrino spectrum. Of course, in a nuclear reactor a very large number of different nuclei and $\beta$ branches contributes to the neutrino spectrum, which makes the task of a first-principles calculation of the neutrino spectrum challenging, and, as we described in Sec. II, theory errors can play an important role, even if one were to assume that the actual nuclear data itself were flawless. The problems of this direct or a priori approach have been explored in detail in Ref. [6], with the conclusion that the inevitable incompleteness of the nuclear databases makes it impossible to account for all neutrinos, reliance on measured total $\beta$ spectra cannot be reduced beyond a certain level, and eventually virtual $\beta$ branches have to be used in addition to the a priori computed spectra.
Neutrino spectra from reactor neutrinos have been obtained by theory calculation by many authors [40-45] and for ${ }^{238} \mathrm{U}$ this is still the only possibility. ${ }^{7}$ The neutrino spectra, which were the state of the art prior to publication of Ref. [6], were obtained by the exclusive use of virtual $\beta$ branches, whose parameters were determined by a fit to measured total $\beta$ spectra [7-9]. This procedure is not independent of input from nuclear databases, either. Here, the information from nuclear databases enters in the form of the empirical mean proton number of the fission fragments as a function of $E_{0}$, which we call the effective nuclear charge $\bar{Z}$, and is computed according to

$$
\bar{Z}\left(E_{0}\right)=\frac{\int_{E_{0}-\Delta / 2}^{E_{0}+\Delta / 2} d E^{\prime} \eta\left(E^{\prime}\right) Z\left(E^{\prime}\right)}{\int_{0}^{\infty} d E^{\prime} \eta\left(E^{\prime}\right)},
$$

with $\Delta$ being an appropriately chosen energy interval.

From a mathematical point of view, the problem at hand is an inversion or unfolding problem: Given the measured total $\beta$ spectrum $\mathcal{N}_{\beta}$, one tries to infer the underlying distribution of $\beta$ branches, $\eta$, which we take to be a continuous distribution over all possible end points ${ }^{8} E_{0}$ :

$$
\mathcal{N}_{\beta}\left(E_{e}\right)=\int d E_{0} N_{\beta}\left(E_{e}, E_{0} ; \bar{Z}\right) \eta\left(E_{0}\right) .
$$

If we neglect the dependence on $\bar{Z}$, problems like this one are known as Fredholm integral equations of the first kind. These problems are ill posed and in general have no unique solution; therefore it is necessary to provide an additional constraint, a so-called regularization scheme. The choice of regularization scheme is essentially an art and not a science, since one has to find the appropriate compromise between finding a reasonably stable solution and the introduction of

\footnotetext{
${ }^{7}$ According to K. Schreckenbach a measurement of the ${ }^{238} \mathrm{U} \beta$ spectrum is currently under way at the FRM II in Garching.

${ }^{8}$ For the moment we neglect the issue of forbidden decays.
} 
an unacceptable bias into the result; for an introduction to the topic of regularized inversion, see, e.g., Ref. [46]. The regularization scheme, which has been developed for our case, relies essentially on averaging the solution over a finite range of $E_{v}$, which corresponds to a top-hat filter. This procedure has been studied in great detail in [47] based on a synthetic data set. The use of a synthetic data set as proxy for real data allows quantification of the size of the bias and thus serves as a valuable tool to fine-tune the regularization scheme. In Ref. [6] it was shown that a $\beta$ spectrum computed from cumulative fission decay yields and the ENSDF database reproduces the measured total $\beta$ spectrum for ${ }^{235} \mathrm{U}$ within an error of about $10 \%$ and therefore can serve as an accurate proxy for the actual data set. We will use a total $\beta$ spectrum derived from the end points contained in the ENSDF database ${ }^{9}$ and we use fission yields for fission by $25 \mathrm{meV}$, i.e., thermal neutrons from the JEFF database, version 3.1.1 [48]. Note, that we neglect the contribution from epithermal and fast neutrons which would change the total spectrum by only a few percent and therefore does not affect its suitability or accuracy as proxy. We also do not apply the small correction for finite irradiation time; this correction can be found in Table VII of Ref. [6]. In computing the resulting $\beta$ spectra we apply the results obtained in Sec. II. We use this data set, which consists of approximately 550 isotopes and contains about 8000 individual $\beta$ branches, to perform a critical study of an inversion purely based on virtual branches.

In Ref. [47] it was shown that, given a precise enough measurement of the total $\beta$ spectrum and accurate knowledge of $\bar{Z}$, the true neutrino spectrum could be recovered to better than within $1 \%$. Unfortunately, several of the assumptions made in this analysis do not apply here: first, the available data were taken with a resolution of $50 \mathrm{keV}$ and second, $\bar{Z}$ can be known only approximately, since the underlying database

\footnotetext{
${ }^{9}$ The end points, branching fractions, assignment of the degree of forbiddeness, and simple $\beta$ spectra, which were computed according to Ref. [6], i.e., without the terms $C, S$, and $G_{v}$, were kindly provided in machine-readable format by the authors of Ref. [6].
}

is incomplete, otherwise there would be no need for the use of virtual branches. Also, the analysis in Ref. [47] does not include the various corrections to the allowed $\beta$-decay shape, and the effect of statistical fluctuations of the actual $\beta$ data was not taken into account. In the following we will quantify the impact of these various factors.

\section{A. Bias and statistical errors}

The basic procedure is to take the measured $\beta$ spectrum, for which, at this stage, we will substitute our simulated $\beta$ spectrum and to start from the highest-energy data point $n_{\beta}^{N}$, where $N$ is the number of data points. Then, one fixes a certain size $s$ of a slice $S$ and takes the data points $S_{1}=$ $\left\{n_{\beta}^{N-s}, n_{\beta}^{N-s+1}, \ldots, n_{\beta}^{N}\right\}$. We then fit an allowed $\beta$ spectrum with a free end point $E_{1}$ and amplitude $a_{1}$ to the data contained in $S_{1}$. We continue this $\beta$ branch to all energies and subtract it from the remaining $N-s$ data points. This procedure is repeated till all data points have been fitted and as result we have a set of $v=N / s$ end points and amplitudes $\left\{E_{i}, a_{i}\right\}$, where $v$ is the number of virtual branches. The set $\left\{E_{i}, a_{i}\right\}$ is a discrete approximation to $\eta\left(E_{0}\right)$ and can be used to compute the neutrino spectrum, by trivially inverting each virtual $\beta$ branch into its corresponding neutrino spectrum. The result of this procedure for ${ }^{235} \mathrm{U}$ with "data" in bins of $50 \mathrm{keV}$ and $s=5$, corresponding to 30 virtual branches, is shown as the green (thin, gray) line in Fig. 2. The thin blue (black) line shows the $\beta$ residuals, which are obviously very small.

The $50 \mathrm{keV}$ antineutrino residuals show oscillations which are due to the simple fact that the $\beta$ spectrum is nonzero at the end point. The amplitude of these oscillations in increased by the inversion procedure, and this increase is a direct consequence of the problem being ill posed in the mathematical sense. To obtain a more regular solution, we can average our result and obtain the thick red line by averaging over $250 \mathrm{keV}$ bins. Clearly, this regularized solution shows oscillations of much smaller amplitude, especially in the region of interest between 2 and $8 \mathrm{MeV}$. We also observe that the remaining oscillations do not average to zero, e.g., between 4

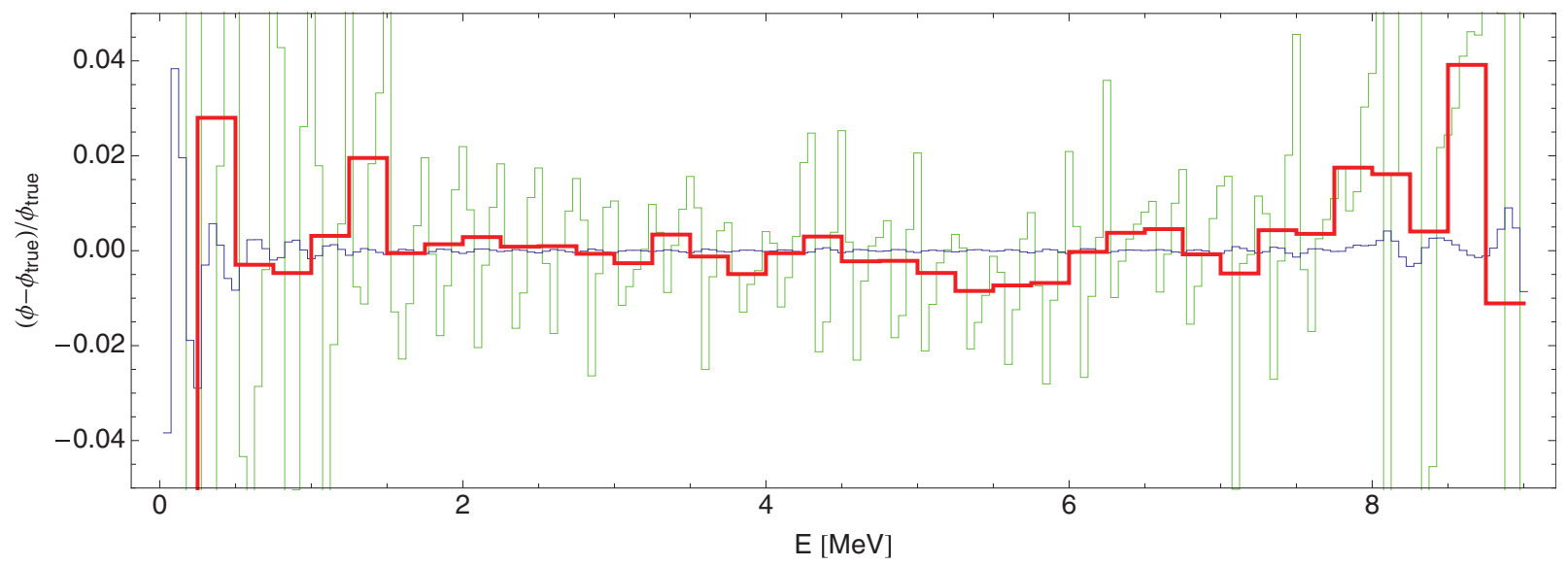

FIG. 2. (Color online) The basic inversion procedure, shown for a synthetic data set for ${ }^{235} \mathrm{U}$. The green (thin, gray) line shows the neutrino residuals in $50 \mathrm{keV}$ bins and the blue (thin, black) line shows the $\beta$ residuals in $50 \mathrm{keV}$ bins. The thick red line depicts the neutrino residuals in $250 \mathrm{keV}$ bins. 


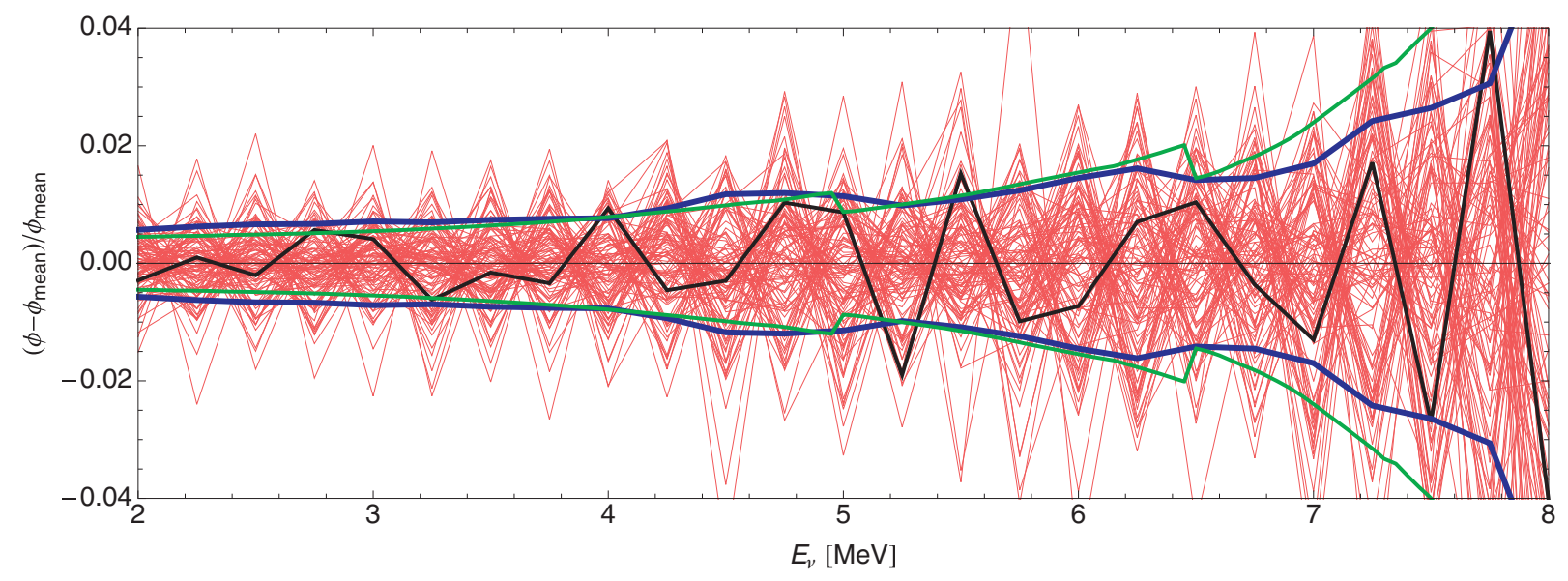

FIG. 3. (Color online) The neutrino flux from the inversion of 1000 random realizations of a synthetic $\beta$ spectrum for ${ }^{235} \mathrm{U}$ relative to the mean outcome of these 1000 trials. The red (thin, gray) lines show a subset of 100 trials. The thick, ragged black line shows one particular example. The smooth thick, blue (black) lines show the standard deviation in each bin, which is the same as the square root of the diagonal elements of the covariance matrix. The dark green (thick, dark gray) lines are the statistical error of the $\beta$ spectrum scaled up by a factor of 7 .

and $6 \mathrm{MeV}$ both the regularized and nonregularized solutions predict a neutrino flux that is too low by about $1 \%$; the result is biased. A finite bias is not surprising and quite common in this type of inversion problem. Using a synthetic data set it is straightforward to quantify the bias by comparing the result of the inversion with the known true flux, as is done in Fig. 2. We, also can vary the starting point of the inversion by removing an increasing number of consecutive data points at the high-energy end and repeat the procedure. The overall features stay the same, but the phase of the oscillations of the nonregularized solution changes gradually, and once we have removed $s$ data points we obtain the initial solution again. This variation of the phase introduces a slight spread in the magnitude of the bias, which we will assign as the error of the bias correction.

The oscillations apparent in Fig. 2 show that the inversion procedure amplifies small perturbations and may be unstable with respect to statistical fluctuations, which in real data are inevitable. Given the statistical errors of the $\beta$ spectra, we can add random fluctuations with the same variance to our synthetic $\beta$ spectra, and for each realization of a randomized $\beta$ spectrum we can perform an inversion. We have done this for 1000 random data sets and the results are shown as red (thin, gray) lines in Fig. 3, where we plot the relative deviation from the mean. The thick black lines show one characteristic example. It is quite obvious that there

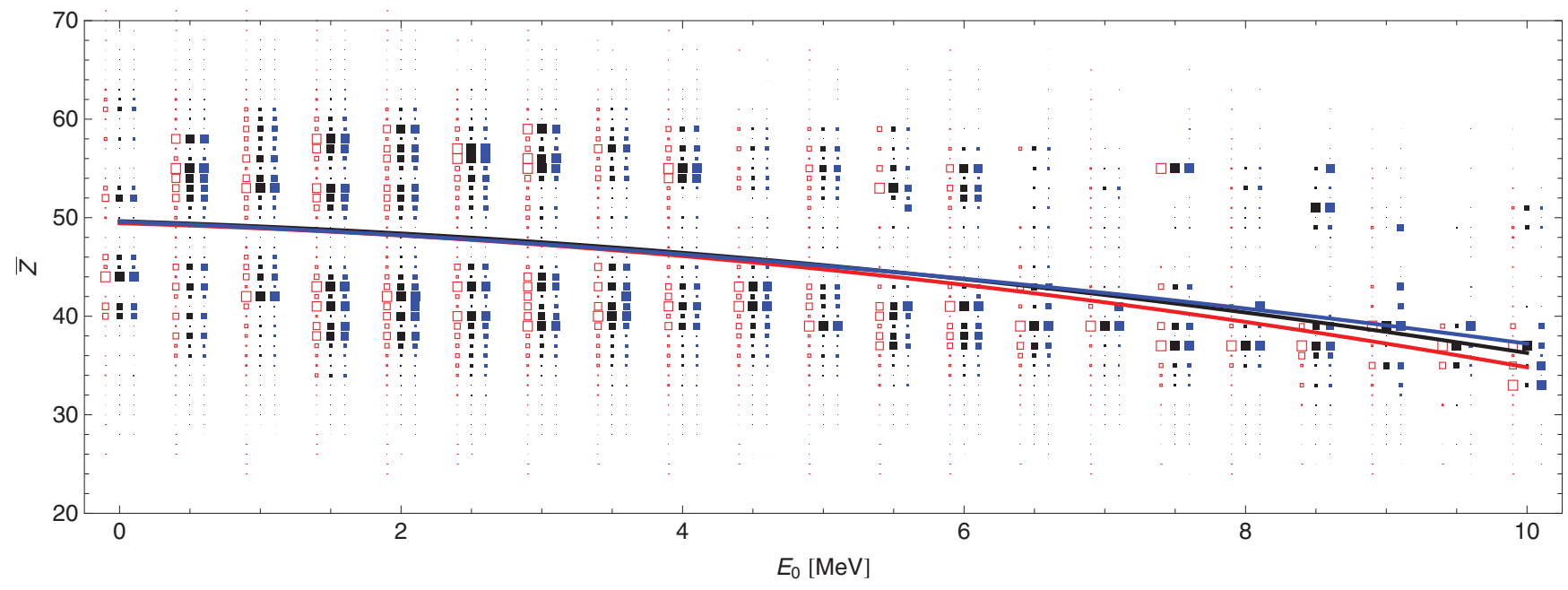

FIG. 4. (Color online) The effective nuclear charge $\bar{Z}$ of the fission fragments of ${ }^{235} \mathrm{U}$ as a function of $E_{0}$. The area of each box is proportional to the contribution of that particular $Z$ to the fission yield in that energy bin. The lines are fits of quadratic polynomials: black, ENSDF database; blue (dark gray), with addition of those isotopes missing from ENSDF by assuming that there is only one $\beta$ branch, each with $E_{0}=Q_{\beta}$; red (light gray), maximum pandemonium as defined in the text. The blue (dark gray, rightmost) filled boxes show the resulting distribution obtained by addition of the missing isotopes with $E_{0}=Q_{\beta}$. The red (light gray, leftmost) empty boxes show the distribution in the maximum pandemonium approximation. 
are very strong anticorrelations between neighboring bins. These anticorrelations are due to oscillations in the inversion procedure, which are excited by statistical fluctuations. Since these oscillations are unphysical and merely a feature of the inversion procedure, we do not want to propagate these oscillations into the final result. The thick, smooth, black line shows the standard deviation in each bin, which turns out to be the same as the square root of the corresponding diagonal element of the covariance matrix of the 1000 randomized inversions. The range delimited by the standard deviation is a good estimate of the statistical error; therefore, we will drop all off-diagonal elements of the covariance matrix. The resulting errors are found to be several times larger in the inverted neutrino spectrum than they are in the underlying $\beta$ spectrum. The errors of the $\beta$ spectrum are shown as dark green (thick, gray) lines but multiplied by a factor of 7 . Note that this scaling factor is not universal and depends sensitively on the details of the inversion, such as the bin width.

\section{B. Effective average nuclear charge}

Finally, we study the degree to which $\bar{Z}$ can be determined and how the incompleteness of nuclear databases affects the inversion procedure. We fit a second-order polynomial in $E_{0}$, where we weight each $\beta$-decay branch by it fission yield $Y_{A, Z}$ and the corresponding branching ratio $b_{A, Z}^{i}$; the result is shown as a black line in Fig. 4 . The black boxes are a two-dimensional histogram of the $Z$ distribution of the fission fragments using the ENSDF database. Note that this distribution is bimodal, because fission proceeds into two asymmetric fragments, and it is therefore quite unexpected that a simple energy-dependent mean $(\bar{Z})$ actually is sufficient to compute accurate neutrino spectra, as previously shown in [47] and in Fig. 2.

We know from comparing the fission yields with the ENSDF database how many and which nuclei are missing, and although we have no detailed information on $\beta$ branches for those nuclei, ENSDF does contain the $Q_{\beta}$ for all of the missing nuclei. Thus, we can try to bracket the effect of the missing nuclei by computing $\bar{Z}$ under two different approximations. First, we assume there is indeed only one $\beta$ branch with $E_{0}=Q_{\beta}$ for each missing isotope. In this case, the effect of the missing isotopes is to add to the high-energy part and increase $\bar{Z}$ by a rather small amount, as shown by the blue (dark gray) curve in Fig. 4. In nuclei far from stability, there will be very many, but individually quite weak, $\beta$ branches, which makes it very difficult to infer the correct level scheme and $\beta$-decay branching fractions from $\gamma$ spectroscopy; this is termed the pandemonium effect [49]. In Ref. [6] the pandemonium effect is taken care of by replacing about 50-100 of the nuclei in the ENSDF database by data that were obtained specifically avoiding the pitfalls of the pandemonium. Here, we would like to use an approximation to this method, which we call the "maximum pandemonium." We take the $17.5 \%$ most neutron-rich nuclei ${ }^{10}$ from the ENSDF

\footnotetext{
${ }^{10} 17.5 \%$ was chosen because the resulting deficit in the total $\beta$ spectra is very similar in magnitude and shape to the one shown in Fig. 5 of Ref. [6].
}

TABLE II. The coefficients for the parametrization of $\bar{Z}$ for the various isotopes. Also given are the ranges as obtained from the maximum pandemonium approximation. $c_{i}$ is the coefficient of $E_{0}^{i}$ in a second-order polynomial in $E_{0}$. Note that in our definition $\bar{Z}$ is the charge of the parent nucleus.

\begin{tabular}{lccc}
\hline \hline Isotope & $c_{0}$ & $c_{1}$ & $c_{2}$ \\
\hline${ }^{235} \mathrm{U}$ & $48.992_{-0.164}^{+0}$ & $-0.399_{-0}^{+0.161}$ & $-0.084_{-0.044}^{+0}$ \\
${ }^{239} \mathrm{Pu}$ & $49.650_{-0.214}^{+0}$ & $-0.447_{-0}^{+0.036}$ & $-0.089_{-0.016}^{+0}$ \\
${ }^{241} \mathrm{Pu}$ & $49.906_{-0.178}^{+0}$ & $-0.510_{-0}^{+0.160}$ & $-0.044_{-0.052}^{+0}$ \\
\hline \hline
\end{tabular}

database and all the $Q_{\beta}$ values of the missing nuclei and replace all their $\beta$ spectra using the following algorithm: We distribute the $\beta$-decay strength evenly between 0 and $Q_{\beta}$ using about ten decay branches. The result is shown as red (light gray) line in Fig. 4. This should yield a reasonable approximation, at least when one averages over a large number of nuclei, as we presently do. The maximum pandemonium approximation is of course rather crude and would not be sufficient for a direct a priori calculation of the neutrino spectrum, but it allows us to gauge the magnitude of the effect of the incompleteness of the nuclear database we use. We repeat this exercise for the other isotopes as well, and the results for $\bar{Z}$ are summarized in Table II.

The induced systematic error is correlated between the isotopes since it has a common physical cause. Note that this is the only place at which information from nuclear databases enters the computation of the neutrino spectrum using only virtual $\beta$ branches. In principle, the nuclear mass $A$ will show a similar change as a function of $E_{0}$, but variations of $A$ have a much smaller impact on the neutrino spectrum and therefore fixing the effective nuclear mass $\bar{A}$ at $\bar{A}=117$ does not lead to any relevant additional bias or error. The value for $\bar{A}$ is chosen based on the average nuclear radius, where the average is determined by weighting each isotope with its cumulative fission yield, and we find a range of about $116-118$ for ${ }^{235} \mathrm{U}$ to ${ }^{241} \mathrm{Pu}$.

\section{APPLICATION TO ${ }^{235} \mathrm{U},{ }^{239} \mathrm{Pu}$, and ${ }^{241} \mathrm{Pu}$}

In this section, we apply the results obtained so far to a direct inversion of the $\beta$ spectra that were measured in the $1980 \mathrm{~s}$ at ILL. We use the $\beta$ spectra for ${ }^{235} \mathrm{U}$ as presented in Ref. [7], for ${ }^{239} \mathrm{Pu}$ from Refs. [8,9], ${ }^{11}$ and for ${ }^{241} \mathrm{Pu}$ from Ref. [9]. The original data for all three isotopes were recorded in $50 \mathrm{keV}$ bins, but unfortunately were published only in $250 \mathrm{keV}$ bins, which is not sufficient for our purposes. However, we were able to obtain finer-binned data from one of the authors [50] of Refs. [7-9]: for ${ }^{235} \mathrm{U}$ we have data in $50 \mathrm{keV}$ bins from 1.5 to $9.5 \mathrm{MeV}$, for ${ }^{239} \mathrm{Pu}$ we have data in $100 \mathrm{keV}$ bins from 1 to $8 \mathrm{MeV}$, and for ${ }^{241} \mathrm{Pu}$ we have data in $100 \mathrm{keV}$ bins from 1.5

\footnotetext{
${ }^{11}$ The actual data on electron spectra are the same in both sources; however, the neutrino spectrum was rederived in the later reference using updated nuclear databases.
} 


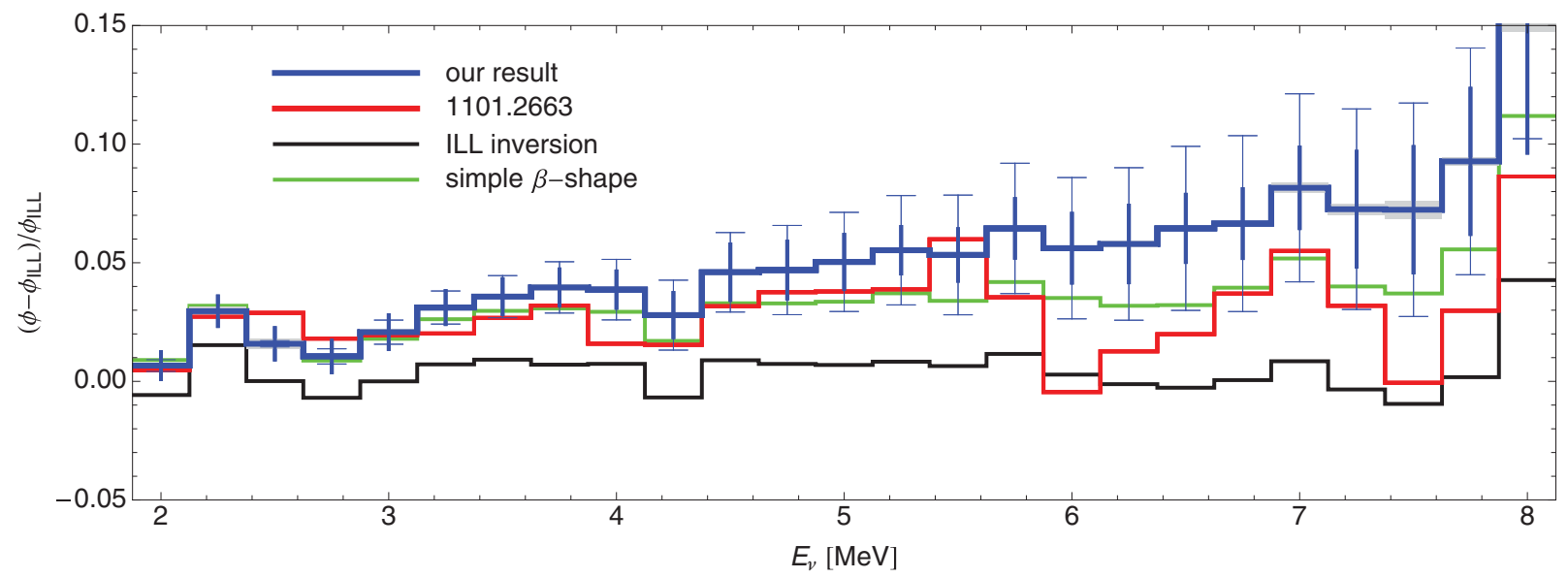

FIG. 5. (Color online) Comparison of our result for ${ }^{235} \mathrm{U}$ with previous inversions, labeled ILL for the results from Ref. [7] and 1101.2663 for the results from Ref. [6]. The thin error bars show the theory errors from the effective nuclear charge $\bar{Z}$ and weak magnetism. The thick error bars are the statistical errors, whereas the light gray boxes show the error from the applied bias correction. The green line, referred to as simple, shows the result if we use the same description of $\beta$ decay as in Ref. [6]. The black line, referred to as ILL inversion, shows our result if we completely follow the procedure outlined in Ref. [7], including the effective nuclear charge given there.

to $9 \mathrm{MeV}$. Thus, we have data for all three isotopes only in the energy interval between 1.5 and $8 \mathrm{MeV}$ and, since the threshold for inverse $\beta$ decay is $1.8 \mathrm{MeV}$ and the results in Ref. [6] range from 2 to $8 \mathrm{MeV}$, we present result only for this interval. Together with the original data we also obtained the size of the statistical error in each bin, which when summed up over $250 \mathrm{keV}$ bins agree with the values published in Refs. [7-9], as do the data themselves. Note that the data on ${ }^{235} \mathrm{U}$ have not only the smallest bin size but also the smallest statistical errors, whereas ${ }^{239} \mathrm{Pu}$ has the largest errors. There is a calibration or normalization error for each data set and we take these values directly from Refs. [7-9] and list them in column 9 of Tables VII-IX.

For each isotope we perform the inversion as outlined in Sec. III but now for the actual data, we use 30 virtual branches with a slice size of 5 for ${ }^{235} \mathrm{U}$ and 23 virtual branches for ${ }^{239} \mathrm{Pu}$ and 25 for ${ }^{241} \mathrm{Pu}$ both with a slice size of 3 . We have tested that variations of the slice size around the values given only mildly affect the results and the chosen values provide the smallest errors around $4 \mathrm{MeV}$. Also, with these choices the energy range of a slice is about the same in all three isotopes and is close to $250 \mathrm{keV}$. By using synthetic data sets for each isotope, we determine the bias and statistical errors; the latter are shown in column 5 of Tables VII-IX, where the square roots of the diagonal elements of the covariance matrix are given. We apply the resulting bias correction, column 3 of Tables VII-IX, to the results extracted from the actual data and assign the spread of biases obtained by varying the starting point of the inversions as error, which is given in column 6 of Tables VII-IX; see also Sec. III A. Then we perform an inversion using the upper and lower ends of the ranges for $\bar{Z}$ from Sec. III B and with the weak-magnetism correction from Sec. IID. The relative differences from the initial inversion result are listed as errors in columns 7 and 8 of Tables VII-IX. Finally the error components in columns 5-9 are added in quadrature and quoted as total error in column 10 of Tables VII-IX.

The total error quoted in Tables VII-IX is only an indication of the actual errors since there are correlations between the various bins. The statistical error is uncorrelated between bins as is the bias error. Both the errors due to $\bar{Z}$ and those due to weak magnetism (WM) are fully correlated between all bins and all isotopes. The normalization errors are fully correlated between bins and between isotopes, since the same apparatus and method to determine the normalization were used for all three isotopes. Because of the asymmetric nature of the errors from $\bar{Z}$ and weak magnetism (WM), they cannot be included exactly in a total covariance matrix. However, already the combined errors form $\bar{Z}$ and WM are close to symmetric and the overall errors are very close to symmetric; thus it is a reasonable approximation to neglect the small asymmetry in errors for most applications.

For completeness, we also provide a parametrization of our results using the familiar exponential of a fifth-order

TABLE III. Result of a fit of a fifth-order polynomial to the logarithm of the flux. The number of degrees of freedom is $25-6$.

\begin{tabular}{lccccccc}
\hline \hline Isotope & $\chi_{\min }^{2}$ & $\alpha_{1}$ & $\alpha_{2}$ & $\alpha_{3}$ & $\alpha_{4}$ & $\alpha_{5}$ \\
\hline${ }^{235} \mathrm{U}$ & 49.3 & 4.367 & -4.577 & 2.100 & $-5.294 \times 10^{-1}$ & $6.186 \times 10^{-2}$ & $-2.777 \times 10^{-3}$ \\
${ }^{239} \mathrm{Pu}$ & 20.8 & 4.757 & -5.392 & 2.563 & $-6.596 \times 10^{-1}$ & $7.820 \times 10^{-2}$ & $-3.536 \times 10^{-3}$ \\
${ }^{241} \mathrm{Pu}$ & 15. & 2.990 & -2.882 & 1.278 & $-3.343 \times 10^{-1}$ & $3.905 \times 10^{-2}$ & $-1.754 \times 10^{-3}$ \\
\hline \hline
\end{tabular}


TABLE IV. These tables compare our result in relation to the fluxes from Refs. [7-9] labeled as $\phi_{\mathrm{ILL}}$ or the inverse $\beta$-decay event rates $R_{\mathrm{ILL}}$. The fluxes and event rates from Ref. [6] are referred to as $\phi_{\mathrm{MLF}}$ and $R_{\mathrm{MLF}}$. For the column labeled "rate" we do not include any normalization errors since they are common to the underlying data; for the column labeled "shape" we assume a free normalization. The significance in the shape column for event rates assumes $10^{6}$ inverse $\beta$-decay events.

\begin{tabular}{|c|c|c|c|c|c|c|c|}
\hline \multirow[b]{2}{*}{${ }^{235} \mathrm{U}$} & \multirow{2}{*}{$\begin{array}{r}\text { shift } \\
\%\end{array}$} & \multicolumn{4}{|c|}{$1 \sigma$ errors [\%] } & \multicolumn{2}{|c|}{$\begin{array}{c}\sigma \\
\text { significance }\end{array}$} \\
\hline & & total & stat & theo. & bias & rate & shape \\
\hline$\phi_{\mathrm{ILL}}$ & 2.4 & 0.7 & 0.1 & 0.6 & 0.1 & 3.7 & 4.5 \\
\hline$R_{\mathrm{ILL}}$ & 3.7 & 1.5 & 0.0 & 1.5 & 0.1 & 2.4 & 3.7 \\
\hline$\phi_{\mathrm{MLF}}$ & 0.4 & 0.7 & 0.1 & 0.6 & 0.1 & 0.6 & 6.0 \\
\hline \multirow[t]{2}{*}{$R_{\mathrm{MLF}}$} & 1.2 & 1.5 & 0.0 & 1.5 & 0.1 & 0.8 & 5.4 \\
\hline & shift & \multicolumn{4}{|c|}{$1 \sigma$ errors $[\%]$} & \multicolumn{2}{|c|}{ significance } \\
\hline${ }^{239} \mathrm{Pu}$ & $\%$ & total & stat & theo. & bias & rate & shape \\
\hline$\phi_{\mathrm{ILL}}$ & 2.9 & 0.7 & 0.3 & 0.6 & 0.2 & 3.9 & 3.2 \\
\hline$R_{\mathrm{ILL}}$ & 4.2 & 1.5 & 0.4 & 1.4 & 0.2 & 2.8 & 3.2 \\
\hline$\phi_{\mathrm{MLF}}$ & $-0.3^{\mathrm{a}}$ & 0.7 & 0.3 & -0.6 & -0.2 & 0.5 & 4.7 \\
\hline \multirow[t]{2}{*}{$R_{\mathrm{MLF}}$} & 1.4 & 1.5 & 0.4 & 1.4 & 0.2 & 1.0 & 4.6 \\
\hline & shift & \multicolumn{4}{|c|}{$1 \sigma$ errors $[\%]$} & \multicolumn{2}{|c|}{ significance } \\
\hline${ }^{241} \mathrm{Pu}$ & $\%$ & total & stat & theo. & bias & rate & shape \\
\hline$\phi_{\mathrm{ILL}}$ & 3.2 & 0.8 & 0.3 & 0.7 & 0.2 & 4.0 & 2.6 \\
\hline$R_{\mathrm{ILL}}$ & 4.7 & 1.6 & 0.2 & 1.5 & 0.2 & 3.0 & 2.6 \\
\hline$\phi_{\mathrm{MLF}}$ & 0.4 & 0.8 & 0.3 & 0.7 & 0.2 & 0.5 & 3.7 \\
\hline$R_{\mathrm{MLF}}$ & 1.0 & 1.6 & 0.2 & 1.5 & 0.2 & 0.7 & 3.7 \\
\hline
\end{tabular}

${ }^{a}$ This difference and its sign are driven by the first bin from $1.875-$ $2.125 \mathrm{MeV}$ and thus the inverse $\beta$-decay rates do not reflect this.

polynomial $[6,51]$ :

$$
\phi_{\nu}\left(E_{v}\right)=\exp \left(\sum_{i}^{6} \alpha_{i} E_{v}^{i-1}\right) .
$$

In performing the fit we follow the description given in Refs. [6,51] but we do not include contributions from the error on $\bar{Z}$ and WM since they are correlated between isotopes and do not change the fit appreciably. The resulting best fit parameters and the minimum $\chi^{2}$ values are given in Table III.

Obviously, the fit for ${ }^{235} \mathrm{U}$ is quite bad, with a $\chi^{2} / \mathrm{dof}$ (degrees of freedom) of more than 2. Also, for all three isotopes the fit parameters are highly correlated and we therefore do not provide any fit errors or correlation matrices, since it seems doubtful whether these could be used to model the errors in the underlying neutrino fluxes. This parametrization should not be used for actual data analysis or error propagation. ${ }^{12}$ Instead, we recommend rebinning our results by using linear interpolation and integrating the resulting fluxes over the new bins. In the same fashion the errors can be rebinned, and we provide results for 250 and $50 \mathrm{keV}$ bins in machine-readable format [52].

\footnotetext{
${ }^{12}$ Nonetheless, this parametrization can be safely used to extrapolate our results to higher energies, since there, the errors of the actual fluxes are sufficiently large to render the inaccuracies of this parametrization harmless.
}

\section{DISCUSSION}

The $\beta$ spectra from Refs. [7-9] have been previously inverted into neutrino spectra and we therefore start by comparing our result with previous results [6-9]. In Fig. 5 we show our result [thick blue (black) line] relative to the results presented in Ref. [7], which is denoted as $\phi_{\mathrm{ILL}}$. We clearly observe that our results point to a significantly enhanced flux with respect to $\phi_{\mathrm{ILL}}$. The results from Ref. [6] are shown as the red (thin, dark gray) line, and up to about $5.5 \mathrm{MeV}$ there is good agreement, whereas at higher energies the result of Ref. [6] exhibits strong oscillation relative to both $\phi_{\mathrm{ILL}}$ and our result, which may be due the coarser binning of the $\beta$ spectra used in Ref. [6]. The thin error bars show the sum of the theory errors, i.e., the effective nuclear charge $\bar{Z}$ and weak magnetism; the thick error bars show the statistical errors; and finally the gray boxes show the error from the bias correction. The theory errors are correlated between all bins; the statistical and bias correction errors are uncorrelated. Not shown is the normalization error, common to all data sets. The thin green line shows our result, if we use the same, simplified, description of the $\beta$-decay spectrum as in Ref. [6] and we see that it is responsible for a sizable fraction of the difference of our result from the others at energies above $5 \mathrm{MeV}$. Finally, the thin black line shows our result if we follow the procedure outlined in Ref. [7], which in particular includes the form of $\bar{Z}$ from [7] and the fact that all corrections to a allowed $\beta$-spectrum shape are applied in an average fashion; we recover the original result quite well. Similar figures can be made for the other isotopes and the results, generally, will be similar, too; the error bars will be much larger owing to the $100 \mathrm{keV}$ binning of the original data and the larger statistical errors in the $\beta$ spectra. As far as the origin of the upward shift in flux relative to the original results [7-9] is concerned, we agree at some level with the comments made in Ref. [6], where at energies below $4 \mathrm{MeV}$ the main effect is ascribed to the branch-by-branch implementation of the various corrections to an allowed $\beta$-spectrum shape. We point out that both an implementation in each virtual branch as well as an implementation in each physical branch in an a priori calculation yield the same effect. Moreover, it is likely that a newly derived average correction based on modern nuclear databases may be nearly as accurate as a branch-by-branch implementation. Of course, derivation of this new average correction would presumably imply an actual study of the corrections first branch by branch, so nothing would be gained. At energies above $5 \mathrm{MeV}$, the new form of the effective nuclear charge $\bar{Z}$ as given in Table II does make up a large fraction of the shift. Note that in contrast to Ref. [6] we do find that an effective nuclear charge is sufficiently accurate and the effect of the actual distribution of $Z$ can be approximated quite well by its properly defined average; this conclusion is supported by the results of Ref. [47]. At high energies the wave function convolution and screening correction to the $\beta$ shape contribute significantly to the overall shift. Once we combine all factors we find about the same upward shift in all isotopes; however we would not characterize it as constant over all energies. 
In Table IV we quantify the mutual agreement or disagreement of the various fluxes and associated inverse $\beta$-decay event rates, where we use the cross section from [53]. Inverse $\beta$ decay is frequently used for the detection of reactor antineutrinos. In this table we list for each isotope the total difference over all energy bins from 2 to $8 \mathrm{MeV}$ of the fluxes from Refs. [7-9] labeled $\phi_{\mathrm{ILL}}$ and the ones from Ref. [6] labeled $\phi_{\mathrm{MLF}}$ from our results. We provide the total error as well as the individual contributions from statistics, theory, and the bias correction. The ratio of the total difference to the total error is what we call the "rate significance," i.e., the significance of the total flux change. We find the total flux change relative to $\phi_{\text {ILL }}$ to be in the range $+(2.4-3.2) \%$. The upward shift in fluxes is significant at the (3.7-4.0) $\sigma$ level. This shift is slightly larger than the one found in Ref. [6]; however, if we compare our total flux with respect to $\phi_{\mathrm{MLF}}$ we find very small differences for ${ }^{235} \mathrm{U}$ and ${ }^{241} \mathrm{Pu}$. For ${ }^{239} \mathrm{Pu}$ the situation is different and we have a lower total flux than $\phi_{\mathrm{MLF}}$, which, however, is entirely due to the first energy bin and could be caused by spurious oscillation in the inversion procedure in either analysis. If we perform the same comparisons for inverse $\beta$-decay event rates, the rows labeled as $R_{\mathrm{ILL}}$ and $R_{\mathrm{MLF}}$, we find that in total event rates the upward shift with respect to $R_{\mathrm{ILL}}$ is a bit larger than for the fluxes at $+(3.7-4.7) \%$, which is due to the relatively large shift at high energies, enhanced by the $E_{v}^{2}$ energy dependence of the cross section. These rate shifts are significant at (2.4-3.0) $\sigma$; the reason for the significance being smaller than for the fluxes is again the larger weight of high energies, which do have a larger shift but also larger errors. As far as total inverse $\beta$-decay rates are concerned, the agreement with the results of Ref. [6] is excellent and no difference reaches more than $1 \sigma$.

In the last column of Table IV we provide the "shape significance," i.e., the square root of the $\chi^{2}$ difference between the various flux and inverse $\beta$-decay rates. For this calculation we assume a free normalization, which is equivalent to subtracting any rate differences. For the shape difference of fluxes we assume infinite statistics, whereas for the inverse $\beta$-decay shape difference we assume $10^{6}$ events. We see that the shape differences can be large and quite significant at even more than $5 \sigma$. This, however, is based only on the flux errors and does not include further complications like experimental errors or errors on the isotope composition of the reactor and burn-up. Thus, at this level of analysis it remains unclear whether an actual neutrino measurement could distinguish between the various flux models.

Apart from the quantitative difference we discussed previously, there are important qualitative differences of our result with respect to earlier inversions. We present, for the first time, a careful and detailed error analysis of both the underlying theory of $\beta$ decays as well as of the inversion procedure itself. We found that most corrections to the statistical $\beta$-decay shape, like finite-size effects, are well understood, and the associated errors are only a fraction of the correction itself; see Sec. IID. However, we also found that the treatment of induced currents in $\beta$ decay is subject to considerable theoretical uncertainty. We studied in detail the example of weak magnetism as the largest contributor and found by comparison with data, that the impulse approximation, which is the basis for assuming that the weak-magnetism correction is universal for all $\beta$ decays, is apparently only accurate within about $100 \%$ for most nuclei and fails completely for a small subset of large-log $f t$ nuclei. For the analysis presented so far we assumed a $100 \%$ error on the weak magnetism corrections, i.e., we neglect the large-log $f t$ nuclei. If we assumed that our limited sample of nuclei were representative for fission fragments, this error would be ten times larger and the sole dominating contribution to the overall error budget, in which case all flux models would be equivalent within these much larger error bars. Even if we assume further that the error bars would remain small, merely adjusting the central value within this larger range to about four times its current value would allow the upward shift to be largely eliminated, and bringing both the calculation presented in Ref. [6] and our result into agreement with the previous ILL fluxes. As a consequence the newfound support for sterile neutrinos as presented in Refs. [10,11] would be weakened considerably.

We also investigated in detail how errors propagate through the inversion procedure and quantified the bias in this method by using synthetic data sets. It is important to realize that the underlying mathematical problem is ill posed and thus we are not dealing with a simple (or complex) fit of a model to data. Therefore, the use of synthetic data sets as first shown in Ref. [47] is essential to gain insight into error propagation and errors inherent in the method. The assumption that the statistical errors of the $\beta$ spectrum would find a one-to-one correspondence in the resulting neutrino spectra is not supported by our Monte Carlo simulations. Also, the size of the bias is non-negligible and would be much larger if we had to use $\beta$ spectra binned in $250 \mathrm{keV}$ bins. The combination of bias and statistical inversion errors from our synthetic data sets yields the total conversion error. We also would like to emphasize that the $\beta$ residuals obtained here are significantly smaller than in Ref. [6] and nowhere exceed the errors of the final result.

In all methods of inversion, input from nuclear databases is required, since the measured $\beta$ spectrum can be fitted by a wide range of different branches by adjusting the nuclear charge. The problem is that nuclear databases tend to be incomplete and may contain significant errors in level assignments, e.g., the pandemonium effect. There are different strategies to deal with these shortcomings, and in Ref. [6] the attempt was made to address these shortcomings directly at the database level by collecting the best available experimental data and supplementing missing data with appropriate theoretical models. This resulted in a database that is about $90 \%$ complete and the remainder was treated by inversion, i.e., with virtual branches. This method, if carefully applied, may have the potential to ultimately yield the most accurate results. Here, we followed a different strategy, which tries to minimize the use of nuclear databases as much as possible. In our inversion procedure, which is solely based on virtual branches, the only place at which information from nuclear databases enters directly is in the form of the effective nuclear charge $\bar{Z}$. Since $\bar{Z}$ is an average quantity, it is relatively straightforward to estimate the impact of both incompleteness, which we know from comparing fission yields with the known entries in the database, and errors in the data themselves, like 
the pandemonium effect. It turns out that $\bar{Z}$ is amazingly robust with respect to these issues and never exceeds the error contributions from nuclear theory. Therefore, we reason that even improved databases would barely change our result.

In combination all our quantified error sources lead to total errors that are close to the ones found previously [6]. At high energies our errors are consistently larger, whereas at lower energies they are either slightly smaller for the case of ${ }^{235} \mathrm{U}$ or slightly larger for the two plutonium isotopes, where "slightly" means of the order of $0.5 \%$.

\section{SUMMARY AND CONCLUSION}

We present a new inversion of the total $\beta$ spectra from fission fragments obtained at ILL [7-9] employing only virtual $\beta$ branches. Our analysis is based on the original data [50], which have finer binning, $50 \mathrm{keV}$ for ${ }^{235} \mathrm{U}$ and $100 \mathrm{keV}$ for ${ }^{239} \mathrm{Pu}$ and ${ }^{241} \mathrm{Pu}$, compared to the published numbers [7-9], but are otherwise identical. In comparison to Refs. [7-9] and [6] we treat the theoretical $\beta$-spectrum shape to a higher level of accuracy by including several subleading corrections, not previously considered in this context. Specifically, we include a more accurate treatment of finite-size effects, screening, and the radiative QED correction for the neutrino; for details see Sec. II D. All of these corrections to an allowed $\beta$ spectrum are applied at a branch-by-branch level, which was not done in Refs. [7-9], where only an average correction was applied. The only point at which nuclear structure information enters this calculation is in the form of the effective nuclear charge $\bar{Z}$, which is derived in Sec. III B. Combining all these ingredients, we find the fluxes as listed in Tables VII-IX. Our results averaged over all energies are in very good agreement with the results found in Ref. [6] and confirm the overall (2-3)\% shift relative to the original inversion [7-9], while at the same time the $\beta$-spectrum residuals are very small, which was not the case in previous analyzes [6].

We put a particular emphasis on evaluating the errors of the final neutrino fluxes. We analyzed the associated theory errors and found that only the contributions from induced currents, for which we studied the weak-magnetism term as the leading contribution, have appreciable theory errors. Our overall theory error is similar to the values previously quoted [6-9]; however, the origin is different; in our case, it is solely due to the difficulties in estimating the size of the weak-magnetism correction. We also point out that there are certain nuclei like ${ }^{14} \mathrm{C}$ in which the weak-magnetism correction is anomalously large due to a suppression of the Gamow-Teller matrix element. A small abundance of nuclei of this kind in fission fragments could enhance the weak magnetism term greatly; see Sec. IID. As a matter of fact, this enhancement could be large enough to account for a large fraction of the flux shift found here and in Ref. [6] and thus may provide a standard model explanation of the reactor anomaly [10] without the need for sterile neutrinos. While we are not advocating this solution to the reactor anomaly throughout this work, it seems that Occam' s razor warrants a closer look into this possibility. We provide an estimate of the effects incompleteness and inevitable inaccuracies of the nuclear structure data have on the determination of the effective nuclear charge $\bar{Z}$; see Sec. III B. We evaluate the inversion errors by using synthetic data sets based on cumulative fission yields and the ENSDF database and use Monte Carlo simulations to "measure" the bias and statistical error. We find that the bias generated by our method is quite small, in agreement with earlier results [47]. The statistical errors are sizable and typically several times larger in the neutrino spectrum than in the underlying $\beta$ spectrum; see Sec. III A. The resulting total errors are similar to previous ones [6] at low energies, but they are larger for the few highest-energy bins. Based on these errors, we find, averaged over all energies, excellent agreement with the results of Ref. [6]; however, if we look at the energy dependence we do find significant shape differences, particular at high energies. These differences should be accessible to measurement, provided a large enough neutrino event sample of the order of $10^{6}$ events can be obtained; see Table IV.

As far as the origin of the shift relative to the original results [7-9] is concerned, we find that both the form of the effective nuclear charge $\bar{Z}$ and the use of an average correction to the allowed $\beta$ shape contribute at a similar level. There are now two independent and complementary analyses, which find the same sign and approximate magnitude of this shift. However, relevant differences between the two analyses do exist, which can be briefly summarized as follows: our analysis uses more finely binned input data and consequently has much smaller $\beta$-spectrum residuals; we use a more detailed treatment of corrections to the allowed $\beta$-spectrum shape and derive the associated errors from this more detailed treatment; we compute the inversion errors, both bias and statistical, based on synthetic data sets; finally, we estimate the errors due to incomplete or incorrect nuclear structure data.

The aforementioned differences make it seem worthwhile to analyze existing reactor data using our results in a spirit similar to Ref. [10] and to investigate whether an actual neutrino spectrum measurement can distinguish between the two calculations [54]. The differences tend to be largest at high neutrino energies, where typically also the largest differences between the neutrino fluxes from the various isotopes are found [51]; thus the impact of these new fluxes and associated errors on neutrino safeguard schemes warrants further study.

\section{ACKNOWLEDGMENTS}

Foremost, I would like to thank K. Schreckenbach for his generosity in making his original data available to me and all the information he provided along with them. I also would like to thank D. Lhuillier and collaborators for their extensive, helpful comments and for making available the $\beta$ end points, branching fractions, and simple $\beta$ spectra derived from the ENSDF database. I need to thank B. Holstein for his clarifications on weak magnetism and induced currents. Furthermore, I would like to acknowledge useful discussions and exchanges with J. Link, L. Piilonen, R. Raghavan, and P. Vogel. This work has been supported by the US Department of Energy under Award No. DE-SC0003915. 
APPENDIX A: SUPPORTING DATA

TABLE V. A reproduction of Table I of Ref. [15].

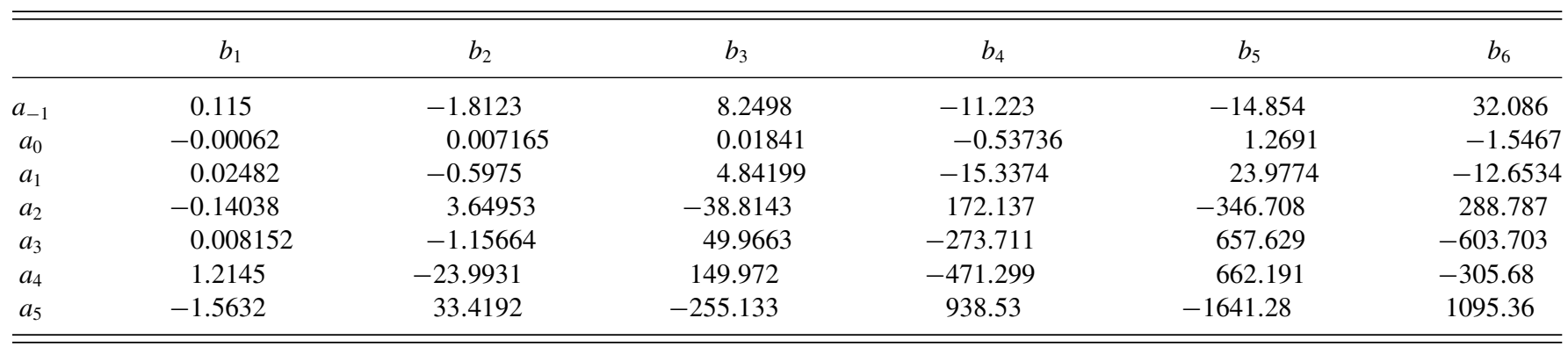

TABLE VI. A reproduction of Table 4.7 of Ref. [16].

\begin{tabular}{cccccccccrr}
\hline \hline$\tilde{Z}$ & 1 & 8 & 13 & 16 & 23 & 27 & 29 & 49 & 84 \\
\hline$N(\tilde{Z})$ & 1.000 & 1.420 & 1.484 & 1.497 & 1.52 & 1.544 & 1.561 & 1.637 & 1.838 & 1.907 \\
\hline \hline
\end{tabular}

APPENDIX B: THE FLUXES

TABLE VII. Results for the ${ }^{235} \mathrm{U}$ antineutrino spectrum. This spectrum corresponds to $12 \mathrm{~h}$ irradiation time. The $\beta$ residuals are given for information only and they do not enter into the computation of the errors. The errors in columns 5 and 6 are fully uncorrelated, whereas the errors in columns 7 and 8 are fully correlated between bins and isotopes. The errors in column 9 are fully correlated between bins and isotopes.

\begin{tabular}{|c|c|c|c|c|c|c|c|c|c|}
\hline $\begin{array}{l}E_{v} \\
(\mathrm{MeV})\end{array}$ & $\begin{array}{r}\beta \text { res. } \\
(\%)\end{array}$ & $\begin{array}{r}N_{\bar{v}} \text { bias } \\
(\%)\end{array}$ & $\begin{array}{c}N_{\bar{v}} \\
\text { (fission }^{-1} \mathrm{MeV}^{-1} \text { ) }\end{array}$ & $\begin{array}{l}\text { Stat. } \\
(\%)\end{array}$ & $\begin{array}{l}\text { Bias err. } \\
(\%)\end{array}$ & $\begin{array}{l}\bar{Z} \\
(\%)\end{array}$ & $\begin{array}{r}\text { WM } \\
(\%)\end{array}$ & $\begin{array}{l}\text { Norm. } \\
(\%)\end{array}$ & $\begin{array}{r}\text { Total } \\
(\%)\end{array}$ \\
\hline 2.0 & 0 & -0.3 & 1.32 & 0.57 & 0.055 & ${ }_{-0.008}^{+0}$ & ${ }_{-0.2}^{+0.14}$ & 1.7 & 1.8 \\
\hline 2.25 & -0.5 & -0.3 & 1.12 & 0.63 & 0.18 & $\begin{array}{l}+0.0074 \\
0\end{array}$ & $\begin{array}{l}+0.017 \\
{ }_{-0.063}\end{array}$ & 1.7 & 1.8 \\
\hline 2.5 & -0.6 & 0.1 & $9.15 \times 10^{-1}$ & 0.66 & 0.21 & ${ }_{0}^{+0.012}$ & $\begin{array}{l}+0.076 \\
-0.11\end{array}$ & 1.7 & 1.9 \\
\hline 3.0 & 0.1 & 0.2 & $6.51 \times 10^{-1}$ & 0.71 & 0.13 & ${ }_{-0.013}^{+0}$ & $\begin{array}{l}+0.35 \\
-0.36\end{array}$ & 1.8 & 1.9 \\
\hline 3.25 & 0 & -0.1 & $5.53 \times 10^{-1}$ & 0.7 & 0.073 & ${ }_{-0.043}^{+0}$ & 0.49 & 1.8 & 2. \\
\hline 3.5 & 0 & -0.2 & $4.54 \times 10^{-1}$ & 0.74 & 0.09 & ${ }_{-0.083}^{+0}$ & $\begin{array}{l}+0.63 \\
{ }_{-0.62}\end{array}$ & 1.8 & 2. \\
\hline 3.75 & -0.3 & 0.4 & $3.64 \times 10^{-1}$ & 0.76 & 0.13 & ${ }_{-0.13}^{+0}$ & $\begin{array}{l}+0.77 \\
{ }_{-0.75}\end{array}$ & 1.8 & 2.1 \\
\hline 4.0 & -0.5 & 0.3 & $2.94 \times 10^{-1}$ & 0.77 & 0.061 & $\begin{array}{l}+0 \\
-0.2\end{array}$ & $\begin{array}{l}+0.91 \\
-0.87\end{array}$ & 1.8 & 2.1 \\
\hline 5.0 & -0.1 & 0.4 & $1.1 \times 10^{-1}$ & 1.1 & 0.047 & ${ }_{-0.56}^{+0}$ & $\begin{array}{l}+1.5 \\
-1.4\end{array}$ & 1.8 & 2.6 \\
\hline 5.25 & 0.2 & 0.4 & $8.64 \times 10^{-2}$ & 0.98 & 0.039 & $\begin{array}{l}-0.00 \\
+0.68\end{array}$ & $\begin{array}{l}-1.4 \\
+1.6 \\
-1.5\end{array}$ & 1.8 & 2.6 \\
\hline 5.5 & -0.1 & 1.0 & $6.46 \times 10^{-2}$ & 1.1 & 0.087 & ${ }_{-0.81}^{+0}$ & $\begin{array}{l}-1.5 \\
+1.6\end{array}$ & 1.8 & ${ }_{-2.8}^{+2.7}$ \\
\hline 5.75 & 0 & 0.7 & $5.1 \times 10^{-2}$ & 1.2 & 0.081 & ${ }_{-0.95}^{+0}$ & $\begin{array}{l}+1.0 \\
-1.9\end{array}$ & 1.8 & $\begin{array}{l}+2.9 \\
-3.9\end{array}$ \\
\hline 6.0 & 0.4 & 0.3 & $3.89 \times 10^{-2}$ & 1.5 & 0.041 & ${ }_{-1.1}^{+0}$ & $\begin{array}{l}+2.0 \\
{ }_{-1.9}\end{array}$ & 1.8 & $\begin{array}{l}+3.1 \\
-3.2\end{array}$ \\
\hline 6.25 & -0.2 & -0.3 & $2.87 \times 10^{-2}$ & 1.6 & 0.14 & $\begin{array}{l}-1.1 \\
+1.3\end{array}$ & +2.2 & 1.9 & $\begin{array}{l}+3.2 \\
+3.4\end{array}$ \\
\hline 6.5 & -0.3 & -0.4 & $2.17 \times 10^{-2}$ & 1.4 & 0.11 & $\begin{array}{l}+0 \\
+1.4\end{array}$ & $\begin{array}{l}+2.3 \\
-2.1\end{array}$ & 1.9 & $\begin{array}{l}+3.4 \\
-3.5\end{array}$ \\
\hline 6.75 & -0.3 & -0.1 & $1.61 \times 10^{-2}$ & 1.5 & 0.078 & $\begin{array}{l}+0 \\
+-1.6\end{array}$ & $\begin{array}{l}+2.4 \\
-2.3\end{array}$ & 1.9 & $\begin{array}{l}+3.4 \\
+3.7\end{array}$ \\
\hline 7.0 & 0.1 & 0.1 & $1.14 \times 10^{-2}$ & 1.7 & 0.22 & $\begin{array}{l}+0 \\
{ }_{-1.8}^{+0}\end{array}$ & $\begin{array}{l}+2.6 \\
-2.4\end{array}$ & 1.9 & $\begin{array}{r}+3.6 \\
-3.9\end{array}$ \\
\hline 7.25 & -0.1 & 0.1 & $7.17 \times 10^{-3}$ & 2.4 & 0.24 & $\begin{array}{l}+0 \\
-2 .\end{array}$ & $\begin{array}{l}+2.7 \\
-2.5\end{array}$ & 1.9 & $\begin{array}{l}+4.1 \\
-4.5\end{array}$ \\
\hline
\end{tabular}


TABLE VIII. Results for the ${ }^{239} \mathrm{Pu}$ antineutrino spectrum. This spectrum corresponds to $3 \mathrm{~h}$ irradiation time. The $\beta$ residuals are given for information only and they do not enter into the computation of the errors. The errors in columns 5 and 6 are fully uncorrelated, whereas the errors in columns 7 and 8 are fully correlated between bins and isotopes. The errors in column 9 are fully correlated between bins and isotopes.

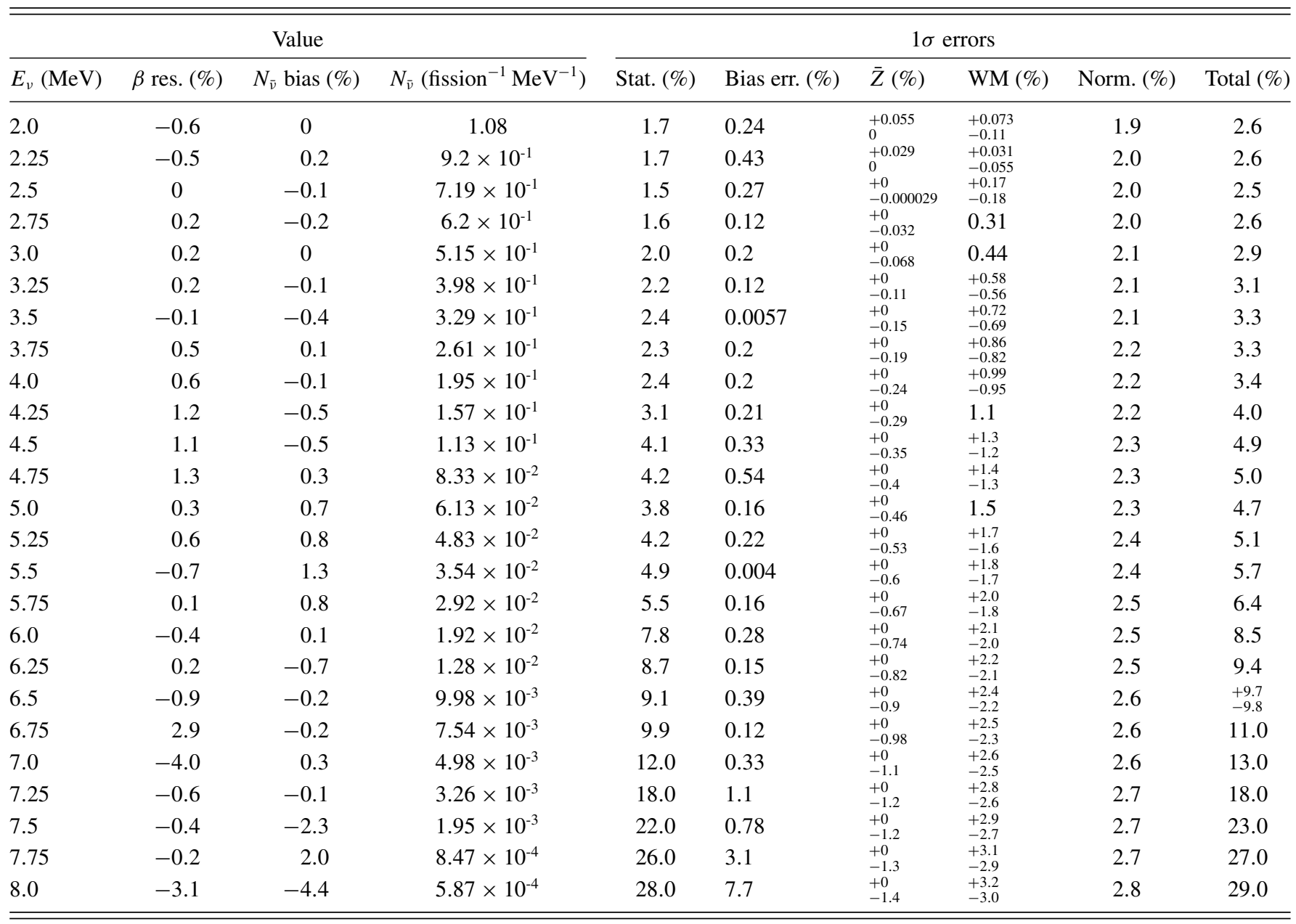

TABLE IX. Results for the ${ }^{241} \mathrm{Pu}$ antineutrino spectrum. This spectrum corresponds to $43 \mathrm{~h}$ irradiation time. The $\beta$ residuals are given for information only and they do not enter into the computation of the errors. The errors in columns 5 and 6 are fully uncorrelated, whereas the errors in columns 7 and 8 are fully correlated between bins and isotopes. The errors in column 9 are fully correlated between bins and isotopes.

\begin{tabular}{|c|c|c|c|c|c|c|c|c|c|}
\hline$E_{v}(\mathrm{MeV})$ & $\beta$ res. $(\%)$ & $N_{\bar{v}}$ bias $(\%)$ & $N_{\bar{v}}\left(\right.$ fission $\left.^{-1} \mathrm{MeV}^{-1}\right)$ & Stat. $(\%)$ & Bias err. $(\%)$ & $\bar{Z}(\%)$ & $\mathrm{WM}(\%)$ & Norm. (\%) & Total $(\%)$ \\
\hline 2.25 & 0 & -0.2 & 1.08 & 1.6 & 0.34 & ${ }_{0}^{+0.13}$ & ${ }_{-0.12}^{0}$ & 1.8 & 2.4 \\
\hline 2.5 & 0.1 & 0.1 & $8.94 \times 10^{-1}$ & 1.4 & 0.33 & ${ }_{0}^{+0.079}$ & ${ }_{-0.23}^{+0.099}$ & 1.8 & 2.3 \\
\hline 2.75 & 0 & -0.3 & $7.77 \times 10^{-1}$ & 1.4 & 0.2 & ${ }_{0}^{+0.024}$ & $\begin{array}{l}+0.24 \\
-0.34\end{array}$ & 1.8 & 2.3 \\
\hline 3.25 & 0 & -0.3 & $5.36 \times 10^{-1}$ & 1.6 & 0.15 & ${ }_{-0.12}^{+0}$ & $\begin{array}{l}+0.52 \\
-0.57\end{array}$ & 1.8 & 2.5 \\
\hline 3.5 & 0 & -0.1 & $4.39 \times 10^{-1}$ & 1.6 & 0.22 & $\begin{array}{l}+0 \\
-0.2\end{array}$ & $\begin{array}{l}+0.65 \\
-0.68\end{array}$ & 1.8 & 2.5 \\
\hline 3.75 & 0 & 0.1 & $3.46 \times 10^{-1}$ & 1.4 & 0.2 & ${ }_{-0.29}^{+0}$ & $\begin{array}{l}+0.79 \\
-0.8\end{array}$ & 1.8 & $\begin{array}{l}+2.4 \\
-2.5\end{array}$ \\
\hline 4.0 & 0 & 0.2 & $2.82 \times 10^{-1}$ & 1.5 & 0.11 & ${ }_{-0.39}^{+0}$ & $\begin{array}{l}+0.93 \\
{ }_{-0.91}\end{array}$ & 1.8 & 2.6 \\
\hline 4.25 & 0 & -0.3 & $2.2 \times 10^{-1}$ & 1.9 & 0.17 & ${ }_{-0.5}^{+0}$ & $\begin{array}{l}+1.1 \\
{ }_{-1}\end{array}$ & 1.9 & 2.9 \\
\hline
\end{tabular}


TABLE IX. (Continued.)

\begin{tabular}{|c|c|c|c|c|c|c|c|c|c|}
\hline 5.25 & 0 & 1.0 & $7.47 \times 10^{-2}$ & 2.3 & 0.14 & $\begin{array}{l}+0 \\
-1\end{array}$ & $\begin{array}{l}+1.6 \\
-1.5\end{array}$ & 1.9 & $\begin{array}{l}+3.4 \\
-3.5\end{array}$ \\
\hline 5.5 & & 1.6 & $5.58 \times 10^{-2}$ & 2.6 & 0.13 & $\begin{array}{l}+0 \\
-1.2\end{array}$ & $\begin{array}{l}+1.8 \\
-1.6\end{array}$ & 1.9 & $\begin{array}{l}+3.6 \\
-3.8\end{array}$ \\
\hline 5.75 & & 0.9 & $4.11 \times 10^{-2}$ & 3.2 & 0.21 & ${ }_{-1.4}^{+0}$ & $\begin{array}{l}+1.9 \\
-1.7\end{array}$ & 1.9 & $\begin{array}{l}+4.2 \\
-4.3\end{array}$ \\
\hline 6.25 & 0.3 & -0.6 & $1.98 \times 10^{-2}$ & 4.4 & 0.33 & ${ }_{-1.7}^{+0}$ & $\begin{array}{l}+2.2 \\
-1.9\end{array}$ & 1.9 & $\begin{array}{r}+5.3 \\
-5.5\end{array}$ \\
\hline 6.5 & -0.1 & -0.3 & $1.54 \times 10^{-2}$ & 4.5 & 0.43 & $\begin{array}{l}+0 \\
-1.9\end{array}$ & $\begin{array}{l}+2.3 \\
{ }_{-2.0}\end{array}$ & 1.9 & $\begin{array}{l}+5.4 \\
-5.7\end{array}$ \\
\hline 6.75 & 0 & -0.1 & $1.09 \times 10^{-2}$ & 4.7 & 0.074 & $\begin{array}{l}+0 \\
-2.1\end{array}$ & $\begin{array}{l}+2.5 \\
-2.2\end{array}$ & 1.9 & $\begin{array}{l}+5.6 \\
-5.9\end{array}$ \\
\hline 7.0 & 0 & 0.6 & $7.75 \times 10^{-3}$ & 4.8 & 0.39 & $\begin{array}{l}+0 \\
-2.3\end{array}$ & $\begin{array}{l}+2.6 \\
{ }_{-2.3}\end{array}$ & 1.9 & $\begin{array}{l}+5.8 \\
-6.1\end{array}$ \\
\hline 7.25 & 0.1 & 0.4 & $4.47 \times 10^{-3}$ & 6.3 & 0.36 & ${ }_{-2.5}^{+0}$ & $\begin{array}{l}+2.7 \\
-2.4\end{array}$ & 2.0 & $\begin{array}{l}+7.2 \\
-7.5\end{array}$ \\
\hline
\end{tabular}

[1] C. L. Cowan, F. Reines, F. B. Harrison, H. W. Kruse, and A. D. McGuire, Science 124, 103 (1956).

[2] C. Bemporad, G. Gratta, and P. Vogel, Rev. Mod. Phys. 74, 297 (2002).

[3] F. Ardellier et al. (Double Chooz Collaboration), arXiv:hep-ex/0606025.

[4] X. Guo et al. (Daya-Bay Collaboration), arXiv:hep-ex/0701029.

[5] J. Ahn et al. (RENO Collaboration), arXiv:1003.1391.

[6] T. A. Mueller et al., Phys. Rev. C 83, 054615 (2011).

[7] K. Schreckenbach, G. Colvin, W. Gelletly, and F. Von Feilitzsch, Phys. Lett. B 160, 325 (1985).

[8] F. Von Feilitzsch, A. Hahn, and K. Schreckenbach, Phys. Lett. B 118, 162 (1982).

[9] A. Hahn et al., Phys. Lett. B 218, 365 (1989).

[10] G. Mention, M. Fechner, T. Lasserre, T. A. Mueller, D. Lhuillier, M. Cribier, and A. Letourneau,, Phys. Rev. D 83, 073006 (2011).

[11] J. Kopp, M. Maltoni, and T. Schwetz, arXiv:1103.4570 [hep-ph].

[12] D. Wilkinson, Nucl. Phys. Instrum. Methods Phys. Res., Sect. A 275, 378 (1989).

[13] E. Fermi, Z. Phys. 88, 161 (1934).

[14] H. Behrens and J. Jänecke, Landoldt-Börnstein Tables, Gruppe I (Springer, Berlin, 1969), Vol. 4.

[15] D. Wilkinson, Nucl. Phys. Instrum. Methods Phys. Res., Sect. A 290, 509 (1990).

[16] H. Behrens and W. Bühring, Electron Radial Wave Functions and Nuclear Beta Decay (Clarendon, Oxford, 1982).

[17] L. R. B. Elton, Nucl. Phys. 5, 173 (1958).

[18] D. H. Wilkinson and B. E. F. Macefield, Nucl. Phys. 232, 58 (1974).

[19] A. Sirlin, Phys. Rev. 164, 1767 (1967).

[20] I. S. Batkin and M. K. Sundaresan, Phys. Rev. D 52, 5362 (1995).

[21] A. Sirlin, Phys. Rev. D 84, 014021 (2011).

[22] M. Gell-Mann, Phys. Rev. 111, 362 (1958).
[23] B. R. Holstein, Rev. Mod. Phys. 46, 789 (1974).

[24] P. Vogel, Phys. Rev. D 29, 1918 (1984).

[25] C. S. Wu, Y. K. Lee, and L. W. Mo, Phys. Rev. Lett. 39, 72 (1977).

[26] F. P. Calaprice and B. R. Holstein, Nucl. Phys. A 273, 301 (1976).

[27] [http://www.nndc.bnl.gov/ensdf/].

[28] D. R. Tilley, C. M. Cheves, J. L. Godwin, G. M. Hale, H. M. Hofmann, J. H. Kelley, C. G. Sheu, and H. R. Weller, Nucl. Phys. A 708, 3 (2002).

[29] F. Ajzenberg-Selove and J. Kelley, Nucl. Phys. A 506, 1 (1990).

[30] D. Tilley, H. Weller, C. Cheves, and R. M. Chasteler, Nucl. Phys. A 595, 1 (1995).

[31] D. Tilley, C. Cheves, J. Kelley, S. Raman, and H. Weller, Nucl. Phys. A 636, 249 (1998).

[32] P. M. Endt, J. Blachot, R. Firestone, and J. Zipkin, Nucl. Phys. A 633, 1 (1998).

[33] C. D. Nesaraja, S. D. Geraedts, and B. Singh, Nucl. Data Sheets 111, 897 (2010).

[34] B. Singh, Nucl. Data Sheets 108, 197 (2007).

[35] E. Browne and J. K. Tuli, Nucl. Data Sheets 111, 1093 (2010).

[36] J. K. Tuli, Nucl. Data Sheets 89, 641 (2000).

[37] A. A. Sonzogni, Nucl. Data Sheets 93, 599 (2001).

[38] F. Ajzenberg-Selove, Nucl. Phys. A 523, 1 (1991).

[39] B. Singh, ENSDF Technical Report, 2004.

[40] K. Takahashi, Prog. Theor. Phys. 45, 1466 (1971).

[41] B. R. Davis, P. Vogel, F. M. Mann, and R. E. Schenter, Phys. Rev. C 19, 2259 (1979).

[42] F. T. Avignone III, and Z. D. Greenwood, Phys. Rev. C 22, 594 (1980).

[43] P. Vogel, G. K. Schenter, F. M. Mann, and R. E. Schenter, Phys. Rev. C 24, 1543 (1981).

[44] H. Klapdor and J. Metzinger, Phys. Lett. B 112, 22 (1982). 
[45] H. Klapdor and J. Metzinger, Phys. Rev. Lett. 48, 127 (1982).

[46] I. J. D. Craig and J. C. Brown, Inverse Problems in Astronomy: A Guide to Inversion Strategies for Remotely Sensed Data (Adam Hilger, Bristol, 1986).

[47] P. Vogel, Phys. Rev. C 76, 025504 (2007).

[48] [http://www.oecd-nea.org/dbforms/data/eva/evatapes/jeff_31 /index-JEFF3.1.1.html].
[49] J. C. Hardy et al., Phys. Lett. B 71, 307 (1977).

[50] K. Schreckenbach (private communication).

[51] P. Huber and T. Schwetz, Phys. Rev. D 70, 053011 (2004).

[52] [http://www.phys.vt.edu/ pahuber/reactorfluxes].

[53] P. Vogel and J. F. Beacom, Phys. Rev. D 60, 053003 (1999).

[54] E. Christensen and P. Huber (unpublished). 